\title{
Coordination and Modulation of Locomotion Pattern Generators in Drosophila Larvae: Effects of Altered Biogenic Amine Levels by the Tyramine $\beta$ Hydroxlyase Mutation
}

\author{
Lyle E. Fox, David R. Soll, and Chun-Fang Wu \\ Department of Biological Sciences, University of Iowa, Iowa City, Iowa 52242
}

\begin{abstract}
Forward locomotion of Drosophila melanogaster larvae is composed of rhythmic waves of contractions that are thought to be produced by segmentally organized central pattern generators. We present a systematic description of spike activity patterns during locomotive contraction waves in semi-intact wild-type and mutant larval preparations. We have shown previously that $T \boldsymbol{\beta} h^{n M 18}$ mutants, with altered levels of octopamine and tyramine, have a locomotion deficit. By recording en passant from the segmental nerves, we investigated the coordination of the neuronal activity driving contraction waves of the abdominal body-wall muscles. Rhythmic bursts of activity that occurred concurrently with locomotive waves were frequently observed in wild-type larvae but were rarely seen in $T \boldsymbol{\beta} h^{n M 18}$ mutants. These centrally generated patterned activities were eliminated in the distal stumps of both wild-type and $T \boldsymbol{\beta} h^{n M 18}$ larvae after severing the segmental nerve from the CNS. Patterned activities persisted in the proximal stumps deprived of sensory feedback from the periphery. Simultaneous recordings demonstrated a delay in the bursting activity between different segments, with greater delay for segments that were farther apart. In contrast, bilateral recordings within a single segment revealed a well synchronized activity pattern in nerves innervating each hemisegment in both wild-type and $T \boldsymbol{\beta} h^{n M 18}$ larvae. Significantly, rhythmic patterns of bursts and waves could be evoked in $T \boldsymbol{\beta} h^{n M 18}$ mutants by head or tail stimulation despite their highly irregular spontaneous activities. These observations suggest a role of the biogenic amines in the initiation and modulation of motor pattern generation. The technique presented here can be readily extended to examine the locomotion motor program of other mutants.
\end{abstract}

Key words: fictive locomotion; biogenic amines; motor pattern; locomotion control; octopamine; tyramine; segmental coordination

\section{Introduction}

Genetic mutations that affect the biogenic amine systems can alter behavior in both invertebrates (Buchner, 1991; Baier et al., 2002; Alkema et al., 2005; Roeder, 2005) and vertebrates (Gainetdinov et al., 2002; Lesch et al., 2003; Holmes et al., 2004). These mutations affect both nonmotor behaviors such as learning (Tempel et al., 1984; Kutsukake et al., 2000; Waddell and Quinn, 2001; Buhot et al., 2003; Murchison et al., 2004) and motor behaviors such as locomotion (Viggiano et al., 2003; Ouyang et al., 2004; Saraswati et al., 2004). Although several systems are amenable to genetic dissection of the biogenic amine systems, little is known about the physiological mechanisms at the level of the neuronal networks that underlie the behavioral deficits produced by these mutations. Our study aims to investigate the effects of identified mutations that are known to produce behavioral deficits on the activity of neuronal networks in larvae of the fruit fly Drosophila melanogaster.

Received Nov. 5, 2005; revised Dec. 14, 2005; accepted Dec. 14, 2005.

This work was supported by National Institutes of Health Grants HD18577 (C.F.W. and D.R.S.) and NS26528 (C.F.W.). We thank Dr. Kalpana White for the $T \beta h^{n M 18}$. We also acknowledge use of the W. M. Keck Dynamic Image Analysis Facility at the University of lowa.

Correspondence should be addressed to Dr. Lyle E. Fox, Department of Biological Sciences, University of lowa, 231 Biology Building, lowa City, IA 52242. E-mail: lyle-fox@uiowa.edu.

DOl:10.1523/JNEUROSCI.4749-05.2006

Copyright $\odot 2006$ Society for Neuroscience $\quad$ 0270-6474/06/261486-13\$15.00/0
Stereotypic behaviors, such as locomotion, are produced by bursts of neuronal activity generated by networks in the CNS known as central pattern generators (CPGs) that can be characterized at an electrophysiological level (Marder and Calabrese, 1996; Grillner, 1999, 2003; MacKay-Lyons, 2002). The Drosophila larval neuromuscular preparation has been well established for studies on synaptic transmission and muscle membrane excitability (Jan and Jan, 1976; Wu et al., 1978; Broadie and Bate, 1993; Kidokoro and Nishikawa, 1994; Fox et al., 2004) and can be used to identify electrophysiological alterations of motor inputs. We used the activity of the motor neurons that innervate bodywall muscles as a measure of the output of the locomotion CPG (Marder and Buchner, 2001). This motor activity is readily recorded from segmental nerves, individual muscle fibers, or synaptic boutons of Drosophila larvae (Budnik et al., 1990; Cattaert and Birman, 2001; Barclay et al., 2002).

In a previous study, we found that Drosophila with a null mutation in the gene that encodes the enzyme tyramine $\beta$ hydroxylase $\left(T \beta h^{n M 18}\right)$, caused by an imprecise excision of a P-element (Monastirioti et al., 1996), have a locomotion deficit (Saraswati et al., 2004). In $T \beta h^{n M 18}$ mutants, octopamine (OA) is reduced and the tyramine (TA) level is increased compared with $T \beta h^{r M 6}$ revertant flies, in which the gene function was restored after P-element excision or to wild-type (WT) flies (Monastirioti et al., 1996; McClung and Hirsh, 1999). To investigate whether 
changes in the patterning or regulation of the CPG underlie the locomotion phenotype of $T \beta h^{n M 18}$ mutants, we established a semi-intact preparation that produces body-wall contractions concurrent with regular bursts of spike activity in WT larvae. Our results demonstrated the tight coordination among CPGs of different hemisegments and the central regulation of rhythmic bursting activity that controls body-wall contractions. We also observed that $T \beta h^{n M 18}$ mutants had fewer rhythmic bursts associated with contraction waves; however, immediately after tactile stimulation, rhythmic bursts could be initiated in the segmental nerves of these larvae. The locomotion phenotype of $T \beta h^{n M 18}$ mutants thus suggests a central role of OA and TA in the initiation and/or modulation of motor pattern generation. The analysis of the locomotion motor program presented here can be readily extended to examine other mutants.

\section{Materials and Methods}

Fly stocks. The WT fly strain, Canton Special (CS), the mutant strain $T \beta h^{n M 18}$, and the revertant $T \beta h^{r M 6}$ were raised on a standard medium at room temperature $\left(23-26^{\circ} \mathrm{C}\right)$. Male third instar $T \beta h^{n M 18}$ larvae were used for the locomotion and physiology experiments. For the other strains, both male and female larvae were tested. $T \beta h^{n M 18}$ and $T \beta h^{r M 6}$ strains were obtained from Dr. K. White (Brandeis University, Waltham, MA).

Video recording of larval locomotion. Video recording of larval locomotion was performed as described previously (Wang et al., 1997, 2002; Saraswati et al., 2004). Wandering third instar larvae were collected from vial walls, washed briefly, and transferred to the center of a fresh $14 \mathrm{~cm}$ Petri dish with a layer of $0.7 \%$ agarose (IBI/Shelton Scientific, Shelton, $\mathrm{CT}$ ) in distilled water. The dish was positioned on an evenly illuminated fluorescent light box $(16 \mathrm{~W})$ situated beneath a video camera (KPC100U; Hitachi, Tokyo, Japan) connected to a video cassette recorder (SLV-696HF; Sony, Tokyo, Japan). The lens aperture was adjusted to capture a high-contrast video image of a dark larval profile against a featureless, bright background. Larvae were allowed to adjust to the Petri dish, and video recording was started only after observing the first stride or wave of contraction. Locomotion of each larva was recorded continuously for at least $2 \mathrm{~min}$ at room temperature $\left(23-26^{\circ} \mathrm{C}\right)$.

Digitization of video recordings and DIAS analysis. Digitization and analysis of video recordings was performed as described previously (Wang et al., 1997, 2002; Saraswati et al., 2004). Briefly, video recordings were digitized at two frames per second on Macintosh G 4 computers (Apple Computer, Cupertino, CA), and larval perimeters were automatically determined from the high-contrast digital movie frames using DIAS software (Soll, 1995) (Soll Technologies, Iowa City, IA). The perimeter stacks were created by overlapping the digitized larval outlines in their relative $x-y$ positions.

Dissection. Feeding or wandering third instar larvae were selected, washed briefly, and pinned onto a glass slide with a shallow well. Larvae were dissected in HL3 saline (Stewart et al., 1994) with a reduced calcium concentration (in mm: $70 \mathrm{NaCl}, 5 \mathrm{KCl}, 0.1 \mathrm{CaCl}_{2}, 20 \mathrm{MgCl}_{2}, 10 \mathrm{NaHCO}_{3}$, 5 trehalose, 67 sucrose, and 5 HEPES, pH 7.2) to minimize muscle contractions. In the majority of experiments, a longitudinal dorsal incision was made, and the cuticle was pinned flat in the recording chamber. Visceral organs were removed to expose the body-wall muscles. The CNS, brain lobes, ventral ganglia, and segmental nerves were left intact unless stated otherwise. The patterned bursting activity recorded from the segmental nerves was not obviously altered when larvae were dissected using a longitudinal lateral incision.

Nerve and focal recording. Spontaneous, ongoing segmental nerve activity was recorded en passant using a suction electrode with an inner diameter of 7-10 $\mu \mathrm{m}$ (Wu et al., 1978) and a differential AC amplifier (P15; Grass Instruments, West Warwick, RI) with bandwidth filters set at 0.1 and $10 \mathrm{kHz}$. Signals were digitized with a Neurocorder (Cygnus Technology, Delaware Water Gap, PA), stored on a VHS-format videocassette recorder (Sony), and simultaneously displayed on a chart recorder (Gould 2400; Gould, Cleveland, OH). The segmental nerve bundles were sucked into the electrode until they formed a loop just inside the tip. These nerve bundles are composed of the segmental and intersegmental nerves that contain most of the motor and sensory axons for a single abdominal body-wall hemisegment. The preparation was then perfused with HL3 saline containing $1.5 \mathrm{~mm} \mathrm{CaCl}_{2}$ at a rate of $\sim 0.5-1 \mathrm{ml} / \mathrm{min}$ unless stated otherwise. Most preparations spontaneously produced contraction waves and bursts of spike activities. The preparations were simultaneously observed visually during nerve recording, and the direction of the contraction waves was documented for the associated bursts. No obvious differences were observed in the bursting activity recorded from the segmental nerves of feeding or wandering larvae. At the end of the experiments, larvae were touched with a silver wire probe $0.1 \mathrm{~mm}$ diameter) on the ventral midline near the head or the tail. Data from larvae that did not respond to the touch and did not have any bursting activity during the entire experiment were discarded.

Isolated brain preparations, consisting of the brain, pharyngeal muscles, and mouth hooks or only the isolated brain, were perfused with HL3 saline with a reduced calcium concentration $(0.1 \mathrm{~mm})$. At higher calcium concentrations ( 0.5 and $1.5 \mathrm{~mm}$ ), motor control-related bursting patterns were masked by a high level of tonic activity.

For focal recordings from the nerve entry points of body-wall muscles (Kurdyak et al., 1994), the electrodes were placed against the muscle fibers without applying suction, and the preparation was perfused with HL3 containing $0.5 \mathrm{~mm} \mathrm{CaCl} 2$ to reduce body-wall contractions. For short intervals $(<15 \mathrm{~min})$, the reduced $\mathrm{Ca}^{2+}$ saline had little effect on the activity recorded from segmental nerves. Longer intervals did eventually reduce the burst frequency and lengthen the burst durations.

Video recording of semi-intact locomotion. Digital video recordings of fictive locomotion were made using a Powershot G6 digital camera (Cannon, Tokyo, Japan) mounted to the eyepiece of the dissecting microscope (Wild, Heerbrugg, Switzerland). Larvae were prepared as described for nerve recording, except that the flow of saline was stopped while recording video to ensure that the bath volume was constant and the larvae remained in focus. Video recordings were saved as QuickTime movies and viewed on a G4 iMac computer (Apple Computer). Individual frames of the larva at different phases of the locomotion cycle were copied from the Quicktime movies and processed using Photoshop Elements (Adobe Systems, San Jose, CA). Quicktime movies and electrophysiological recordings were synchronized by signals generated by lightly tapping the table.

\section{Results}

\section{Locomotion and Motor patterns of $\boldsymbol{T} \boldsymbol{\beta} \boldsymbol{h}^{n M 18}$ mutant and control larvae}

$T \beta h^{n M 18}$ mutant larvae have been shown to have a locomotion deficit that is most likely associated with a lesion in the $T \beta h$ locus (Saraswati et al., 2004). Locomotion patterns for $T \beta h^{n M 18} \mathrm{mu}$ tant larvae, the $T \beta h^{r M 6}$ revertants, and CS wild type are shown in Figure 1. Larval crawling, as shown in contour stacks, can be divided into two clear components, forward locomotion and pausing (cf. Wang et al., 1997, 2002). Forward crawling is composed of alternating waves of body-wall contractions and elongation. The pausing episodes consist of lateral movement of anterior segments without forward locomotion (Fig. 1) (cf. Wang et al., 1997, 2002; Saraswati et al., 2004). $T \beta h^{n M 18}$ mutants that have increased TA levels and reduced OA levels (Monastirioti et al., 1996) have a much shorter path than the $T \beta h^{r M 6}$ revertant or WT larvae that have normal levels of OA and TA (Monastirioti et al., 1996). The most significant difference between $T \beta h^{n M 18}$ mutants and WT larvae is the time spent in forward locomotion, only $\sim 35 \%$ for $T \beta h^{n M 18}$ mutants compared with $\sim 80 \%$ for WT larvae (Saraswati et al., 2004). This behavioral phenotype of the $T \beta h^{n M 18}$ mutants could be attributable to changes in the activity of the central neurons that generate the motor program and/or changes in the properties of the body-wall muscles (Kutsukake et al., 2000; Nagaya et al., 2002) that produce the motor output. We hypothesized that the locomotion deficit is primarily caused by 

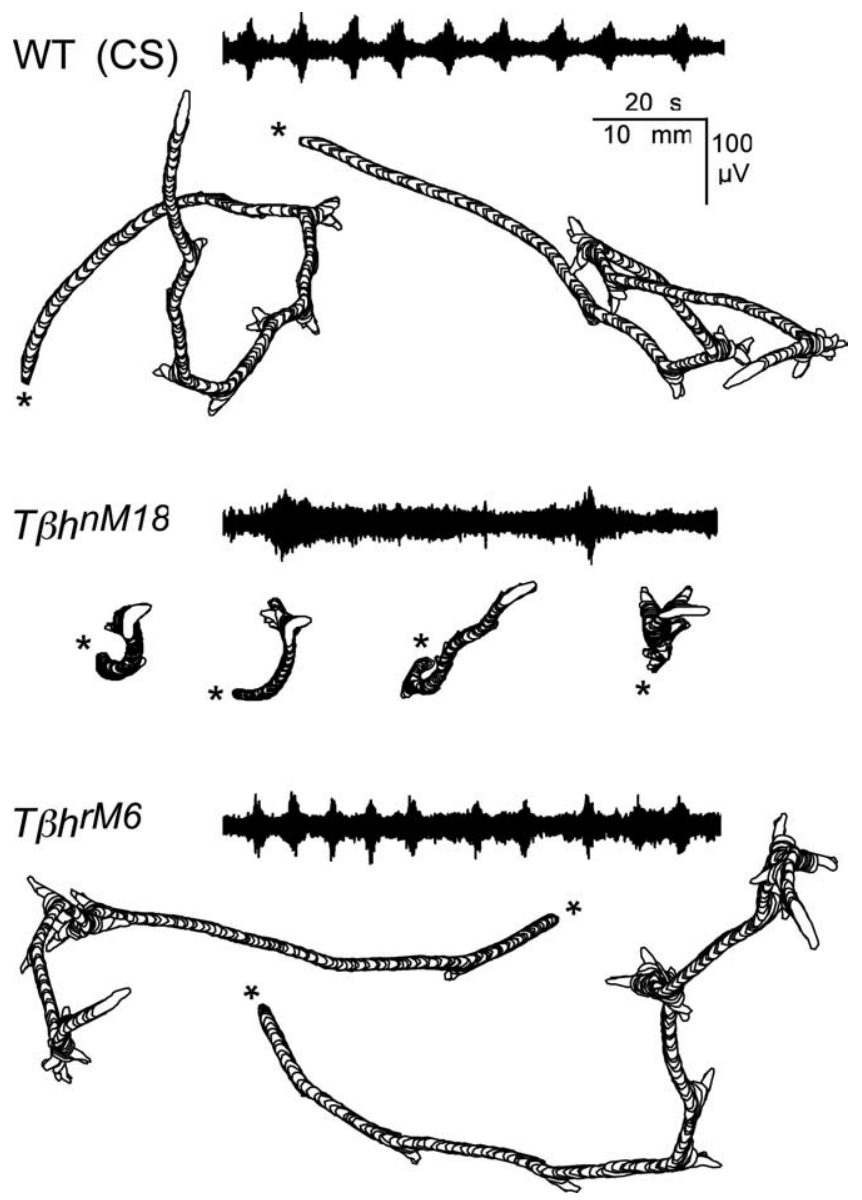

Figure 1. Representative crawling patterns and segmental nerve activity of WT, $T \beta h^{r M 6}$, and $T \beta h^{n M 18}$ Drosophila larvae. Larval position and outlines were captured by video recording for a duration of $2 \mathrm{~min}$, digitized at $2 \mathrm{fps}$, and depicted by perimeter stacks. $T \beta h^{n M 18}$ mutant larvae traveled less distance than WT or $T \beta h^{r M 6}$ revertant larvae. The distance traveled by WT and $T \beta h^{r M 6}$ larvae were similar. The beginning of locomotion is marked by an asterisk. En passant recordings made from the nerve innervating abdominal segment 3 from semi-intact preparations of larvae in the wandering stage are shown. Activity recorded from WT and $T \beta h^{r M 6}$ nerves was organized into regular rhythmic bursts, whereas recordings from $T \beta h^{n M 18}$ mutants showed a high level of tonic nerve activity with very few bursts.

changes in the activity of neurons that constitute the locomotive CPG.

The body-wall musculature of Drosophila larvae is organized in a segmentally repeated pattern (Fig. $2 A$; see Fig. 7A). Abdominal hemisegments $2-7$ are composed of $\sim 30$ muscle fibers that can be identified based on their orientation and position (Crossley, 1978). Many of the motor neurons that innervate the muscles have been identified and are located in the ventral ganglia, with their axons projecting through the segmental nerves to innervate their target muscle fiber(s) (Sink and Whitington, 1991; Landgraf et al., 1997; Hoang and Chiba, 2001). We recorded the activity of the motor neurons that innervate body-wall muscles en passant from segmental nerves as a measure of the output of central neurons (Wu et al., 1978; Cooper and Neckameyer, 1999; Marder and Buchner, 2001; Barclay et al., 2002).

It was evident that the activity recorded from the segmental nerves of $T \beta h^{r M 6}$ and WT larvae was organized into regular rhythmic bursts (Fig. 1). As a measure of regularity of this rhythmic activity, we counted the number of larvae that had spontaneous bouts of activity that consisted of at least three bursts within $30 \mathrm{~s}$ for WT and $T \beta h^{r M 6}$ larvae. Bouts were observed in $88 \%$ of the $T \beta h^{r M 6}$ larvae (7 of 8 larvae) and $85 \%$ of the CS larvae (17 of 20 larvae). In contrast, regular rhythmic activity was much less common for $T \beta h^{n M 18}$ mutants with only $25 \%$ (5 of 20 larvae) showing spontaneous bursting activity. Instead, there was a high level of tonic nerve activity with very few bursts. In addition, we observed that bursts from all three genotypes had a similar spindle shape with larger amplitude neurons firing near the end of the bursts. These nerve recordings are consistent with the hypothesis that the locomotion deficit in $T \beta h^{n M 18}$ mutants is attributable to a defect in motor pattern generation. Thus, we investigated the motor output in more detail.

Nerve activity is correlated with waves of muscle contractions In Drosophila, motor neurons fire in rhythmic bursting patterns that can be changed by pharmacological agents or genetic manipulations (Budnik et al., 1990; Cooper and Neckameyer, 1999; Cattaert and Birman, 2001; Barclay et al., 2002; Suster et al., 2004; Wang et al., 2004). However, little is known about the bilateral and intersegmental coordination of bursting patterns in Drosophila larvae. Previous studies have focused on the activity of a subset of neurons innervating a specific muscle fiber or recorded from a single nerve. We set out to examine bilateral and intersegmental coordination of neuronal activity by simultaneous en passant recording from pairs of segmental nerves with suction electrodes.

Forward locomotion of $D$. melanogaster larvae is produced by rhythmic waves of contractions that proceed from posterior to anterior body segments. We wanted to determine the relationship between the spontaneous rhythmic activity recorded from the segmental nerves and the waves of body-wall contractions. Semi-intact WT larvae were used for simultaneous video recordings of muscle contractions and electrophysiological recordings of nerve activity (Fig. 2). We found that, in the HL3 saline, dissected larvae with the CNS intact could stably maintain regular rhythmic waves of muscle contractions for tens of minutes.

Selected frames from a video recording show a wave that began in the posterior segments and smoothly progressed to the anterior segments (Fig. 2C). Contractions were preceded by an increase in neuronal activity that began in the nerve innervating posterior segment 7 and progressed to the nerve innervating anterior segment 3 (Fig. 2 D). No waves of muscle contractions were observed in larvae when the segmental nerves were cut. These results suggest that the rhythmic nerve activity is important for the regular contraction waves observed in the semi-intact preparation and possibly even for larval crawling. Notably, rhythmic bursting activity and contraction waves were rare in $T \beta h^{n M 18}$ mutants.

\section{Bilateral and intersegmental coordination of neuronal activity in WT larvae}

The coordination of the bursting activity during regular rhythmic waves of body-wall contractions was compared bilaterally within a single segment and unilaterally between anterior and posterior segments for WT larvae (Fig. 3). A representative simultaneous bilateral recording is shown for a single segment, abdominal 5, that reveals a synchronized pattern of activity in nerves innervating each hemisegment (Fig. 3A). Similar coordination of both right and left nerves was also observed for abdominal segments 3-7. The level of grouped activity detected in the segmental nerve sometimes varied between the nerves for each hemisegment. This waveform variation was probably caused by geometric factors that shape the unit activities of different axons (e.g., the size of the 
A
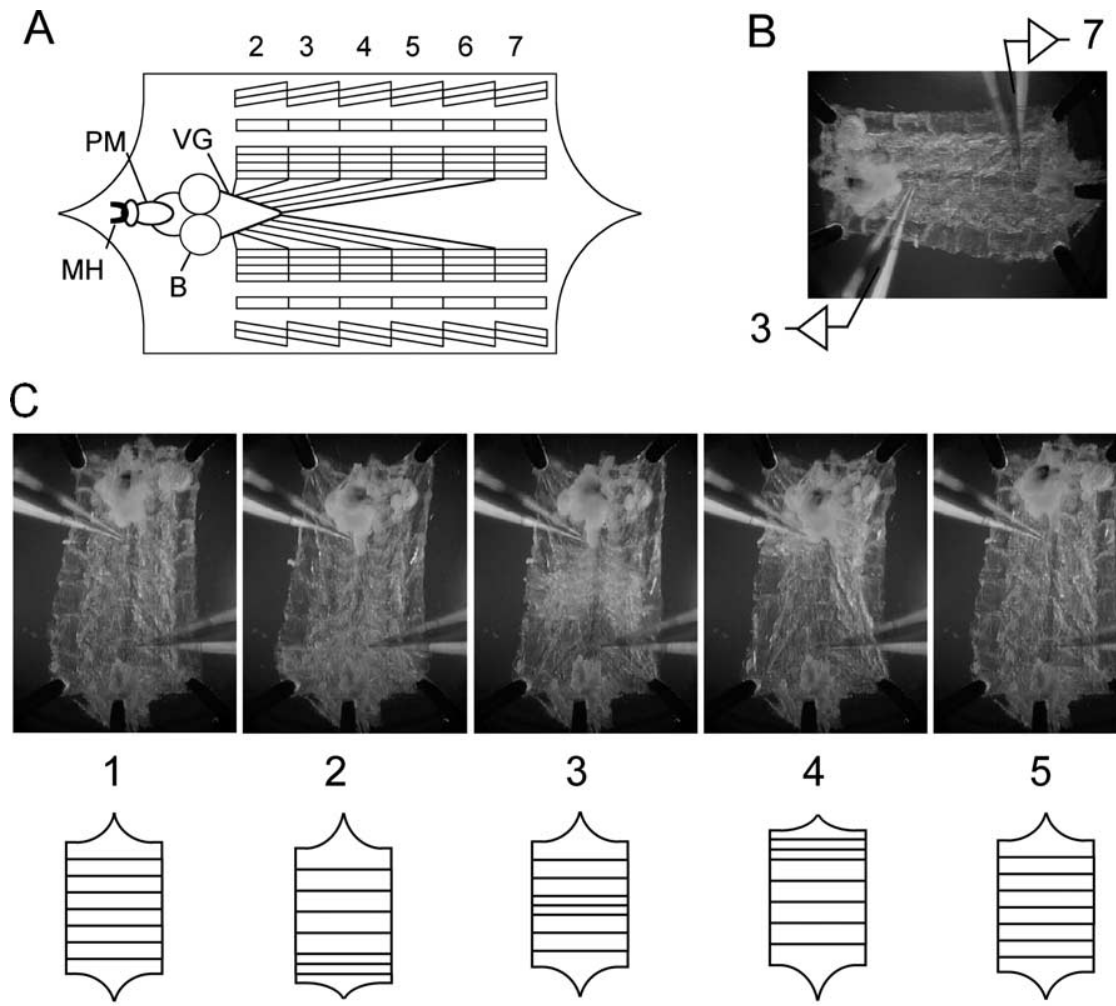

4

D

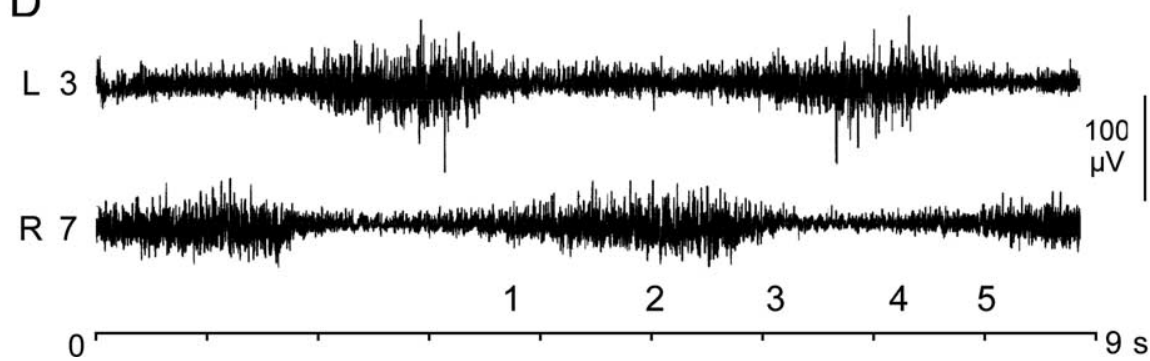

Figure 2. Correlation of nerve activity with waves of muscle contractions in WT larvae. A, Diagram of Drosophila larval neuromuscular preparation. Larval brain hemispheres (B), ventral ganglia (VG), pharyngeal muscles (PM), mouth hook (MH), and innervation of segmental body-wall muscles are indicated. Note the stereotypic pattern of the body-wall muscles in abdominal segments 2-7. B. Photograph of semi-intact Drosophila larval neuromuscular preparation showing en passant suction electrode placement. A recording from abdominal segmental nerves 3 and 7 is as indicated. C, D, Dissected larvae with the CNS intact produced regular rhythmic waves of muscle contractions during simultaneous video recordings and electrophysiological recordings. C, Selected frames from a video recording are in the top panel, and waves are diagramed in the bottom panel. In the initial phase of the contraction cycle, all of the body-wall muscles were relaxed (1), then the posterior segments were contracted (2). The contraction proceeded toward the head in a wave with medial (3) and anterior (4) segments contracting. Finally, all of the muscles relaxed before the next wave (5). $\boldsymbol{D}$, Contractions were preceded by an increase in neuronal activity recorded from the segmental nerves that innervate the segment. Bilateral recordings were from left abdominal hemisegment 3 (L 3 ) and right hemisegment 7 (R 7). The time reference to contraction phases (1-5) during the cycle is indicated.

nerve loop drawn into the suction electrode) and did not interfere with the identification of bursts.

Activity recorded unilaterally from nerves innervating anterior and posterior segments also had similar patterns and were well coordinated. There was, however, a phase delay in the peak bursting activity recorded from nerves innervating anterior and posterior segments (Fig. 3B). Three different patterns of bursting activity were observed during simultaneous recordings from nerves innervating anterior and posterior segments (Fig. $3 B$ ). The most common pattern was associated with regular rhythmic body-wall contractions that originated in posterior segments and propagated to anterior segments in a wave ( $\mathrm{P}$ to A wave). During $\mathrm{P}$ to A waves, neuronal activity was detected first in nerves inner-
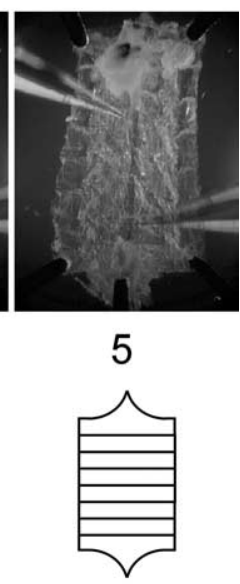

vating posterior segments and then, after a delay, it was detected in anterior nerves. The duration of the bursts for the $\mathrm{P}$ to $\mathrm{A}$ waves tended to be longer for the nerves innervating the posterior segments (Fig. $3 B$ ). The other common rhythmic pattern was associated with regular waves of contractions that initiated in anterior segments and propagated to posterior ones (A to $P$ waves). The properties of $A$ to $P$ waves were opposite of those for $\mathrm{P}$ to A waves in that neuronal activity was longer for nerves innervating anterior segments where A to $\mathrm{P}$ waves originated (Fig. $3 B$ ). We also observed nonrhythmic nerve activity that was not associated with regular waves of body-wall contractions (Fig. 3B). Several different activity patterns were categorized as nonrhythmic: periods of tonic spiking activity and bursts that were not associated with visible contractions, bursts associated with nonpropagating regional muscle fiber twitches, and bursts that were associated with a single contraction wave. Notice that the burst durations and interburst intervals were highly irregular for the nonrhythmic nerve activity. Individual larvae often spontaneously switched between the three patterns (Fig. 3B).

The cycle period was determined for episodes of locomotion. Data were collected from bilateral recordings within a single segment and unilateral recordings between anterior and posterior segments from preparations that maintained rhythmic activity lasting at least 11 bursts. The period was measured among activity peaks from adjacent bursts, yielding a cycle period of $6.5 \pm 0.1 \mathrm{~s}$ (average \pm SEM) for the 440 bursts pooled from both electrodes of 20 animals. It was not significantly different for the right or left sides from bilateral recordings (right and left, $7.2 \pm 0.3 \mathrm{~s} ; n=$ 10 ; paired $t$ test, $p=0.47$ ) or for anterior segment 3 and posterior segment 7 from unilateral recordings (anterior, $5.7 \pm 0.2 \mathrm{~s}$; posterior, $5.8 \pm 0.2 \mathrm{~s} ; n=10$; paired $t$ test, $p=0.32$ ). The cycle period varied considerably for different larvae and for different bouts selected from an individual larva, ranging from $3.0 \pm 0.1 \mathrm{~s}$ to $14.1 \pm 0.2 \mathrm{~s}$. The cycle period was longer for nerve recordings from the semi-intact preparation than for contraction waves from freely crawling larvae that have a reported cycle period of $1.0 \pm 0.02 \mathrm{~s}(n=47)$ (Saraswati et al., 2004). Longer cycle periods are commonly observed in other reduced vertebrate and invertebrate preparations (Friesen and Cang, 2001).

In addition, we observed that the delay between bursts recorded from the segmental nerves increased as the electrodes were moved farther apart. Figure $4 A$ demonstrates the segmental delay from a single larva. Nerve activity was initially recorded bilaterally during regular $\mathrm{P}$ to A waves of contractions. Then one of the electrodes was moved to other more posterior segments. 

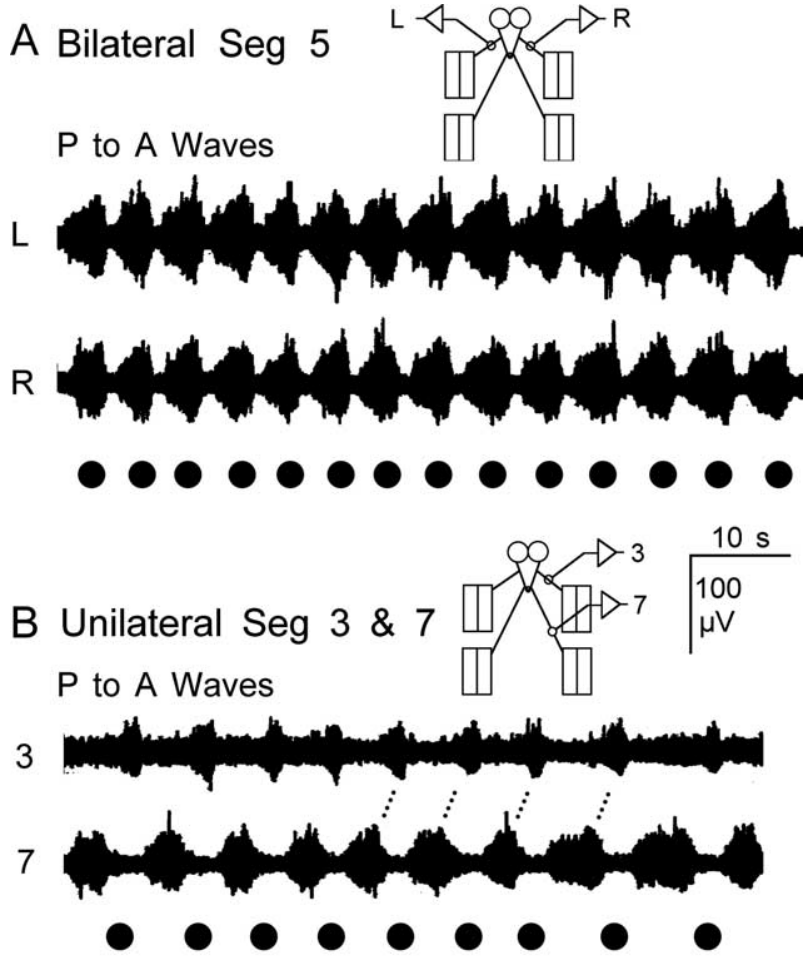

A to P Waves

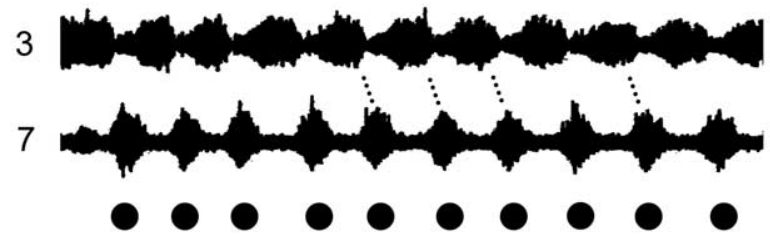

Nonrhythmic

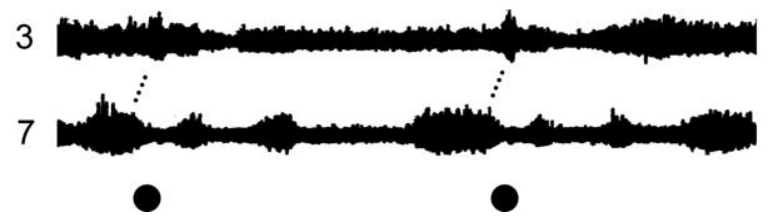

Figure 3. Bilateral and intersegmental coordination of neuronal activity recorded from segmental nerves of WT larvae. $A$, En passant recordings made bilaterally from segmental nerves innervating segment 5 . Synchronized bursting activity was observed from the right $(R)$ and left (L) sides associated with waves of contractions. $\boldsymbol{B}$, En passant recordings made unilaterally from nerves innervating anterior segment 3 and posterior segment 7 . There was a phase delay in activity recorded from nerves innervating anterior and posterior segments for unilateral recordings. Three common patterns of activity were observed in unilateral recordings: rhythmic activity recorded during anterior to posterior propagating contraction waves, rhythmic activity recorded during posterior to anterior waves, and nonrhythmic bursts unassociated with regular contraction waves. Recordings were from a single larva that spontaneously switched between the patterns. Note that the bursts tend to be longer in the body region where the wave originates. Muscle contraction waves here and in the following figures are marked with black dots. Seg, Segment.

The peak activity was simultaneous in the bilateral segmental nerve 3 recordings. However, activity recorded from segmental nerve 5 preceded the activity from segmental nerve 3 , and the interval was increased further in recordings from segmental nerve 7 (Fig. 4A). Delays were also observed for A to P contraction waves. In the example shown in Figure $4 B$, segmental nerve 3 activity preceded that of segmental nerve 7 .
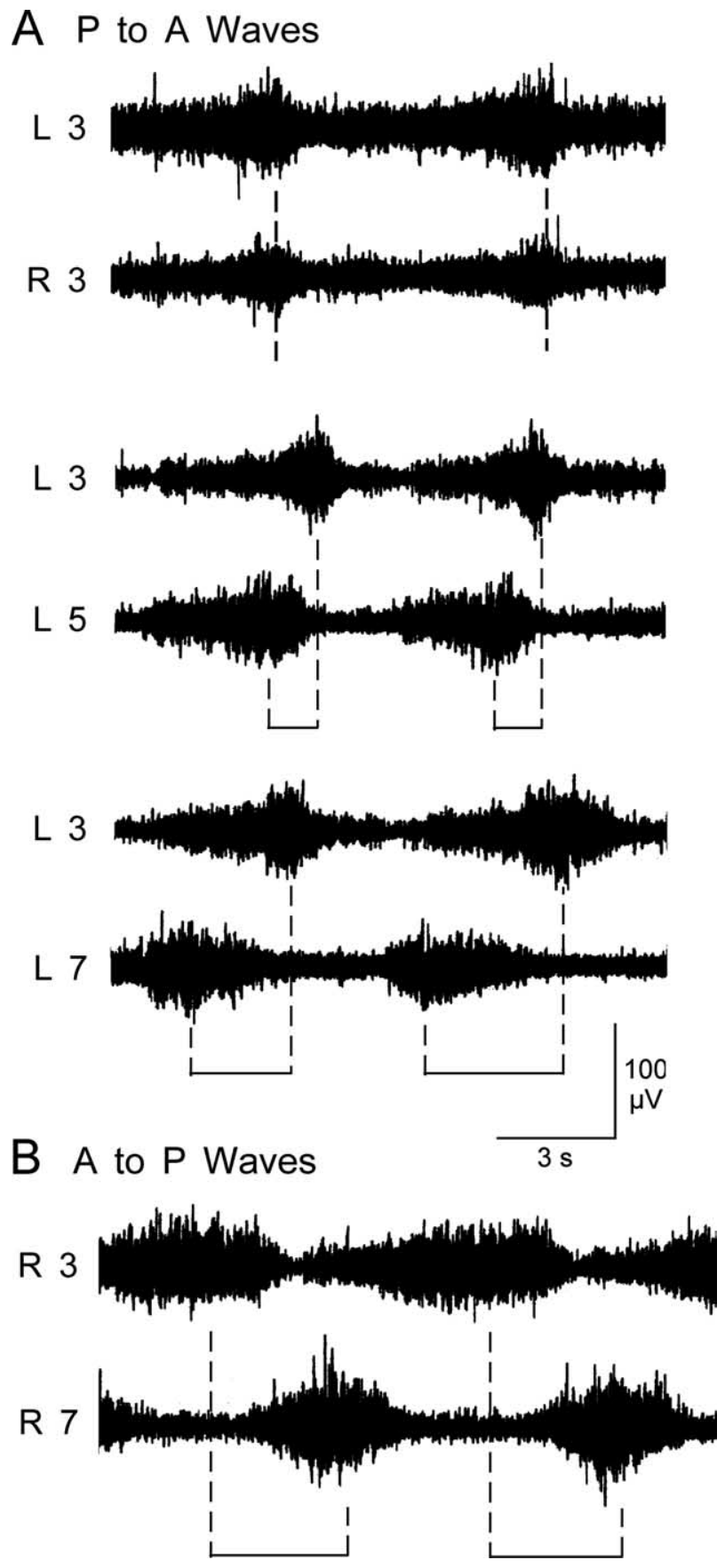

Figure 4. Delay between bursts recorded from anterior and posterior segmental nerves. $A$, Nerve activity was recorded bilaterally during regular posterior to anterior waves of contractions, and the electrode was moved to other more posterior segments within a single larva. The peak activity was simultaneous in the bilateral segmental nerve 3 recordings (top). Activity recorded from segmental nerve 5 preceded the activity from segmental nerve 3 (middle), and the interval was increased further in recordings from segmental nerve 7 (bottom). $\boldsymbol{B}$, Segmental delay during an anterior to posterior locomotive wave. Activity recorded from segmental nerve 3 preceded the activity from segmental nerve 7 . L, Left; $R$, right.

Isolated CNS is capable of generating patterned segmental nerve activity

The segmental nerves contain the axons of the ventral ganglia motor neurons that innervate the body-wall muscles as well as the axonal projections from peripheral sensory neurons. We examined whether depriving sensory feedback could drastically alter the patterned neuronal activity by cutting the segmental nerves 


\section{A Nerves Intact}
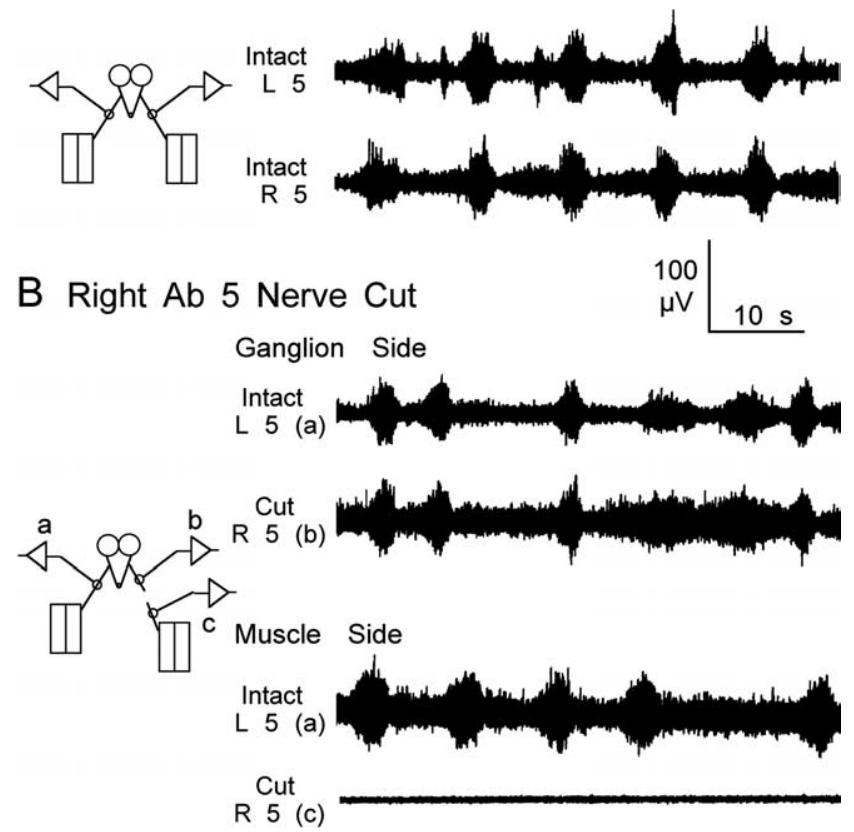

C Both Ab 5 Nerves Cut
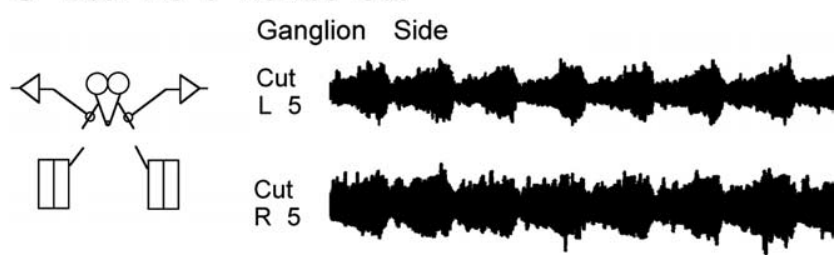

Figure 5. Central origins of rhythmic activity recorded from segmental nerves of WT larvae. $A$, Activity recorded bilaterally from segment 5 with both nerves intact was coordinated and in phase. $\boldsymbol{B}$, Cutting the nerve had little effect on the bursting activity recorded from the severed nerve attached to the ventral ganglion, whereas activity recorded from the nerve connected to the body wall ceased. C, Bursting activity was still coordinated and in phase even after bilaterally cutting the nerve to segment 5 . $\boldsymbol{A}$ and $\boldsymbol{B}$ were from the same larva, and $\boldsymbol{C}$ is a representative example from a different larva. $L$, Left; $R$, right; $A b$, abdominal.

and recording from their stumps. When all of the nerves were intact, a similar bursting pattern was observed during bilateral nerve recordings, as shown in Figure 5, for both nerves innervating segment 5. After cutting the nerve to the right side, the rhythmic bursting activity continued in the nerve stump connected to the ventral ganglion. In contrast, no activity was detected in the stump connected to the body wall. This suggests that the spontaneous activity observed in the en passant nerve recordings mostly originates in the ventral ganglion and reflects the activity of motor neurons.

The segmental nerves were sequentially cut to determine whether peripheral input was required for generating the motor pattern. Cutting the nerves bilaterally to an individual segment (Fig. $5 C$ ) or cutting the nerves to all of the abdominal segments on one side (Fig. 6A) had little effect on the activity recorded from segmental nerves. Burst-like activities were observed even after all of the abdominal nerves on both sides were cut, but the bursts were not as regular (Fig. 6B). The temporal sequence in burst-like activities between anterior and posterior segmental nerves was still preserved after cutting abdominal nerves. We eliminated most peripheral input by cutting the remaining thoracic nerves to separate the brain from the body wall. We found
A Right Ab Nerves Cut
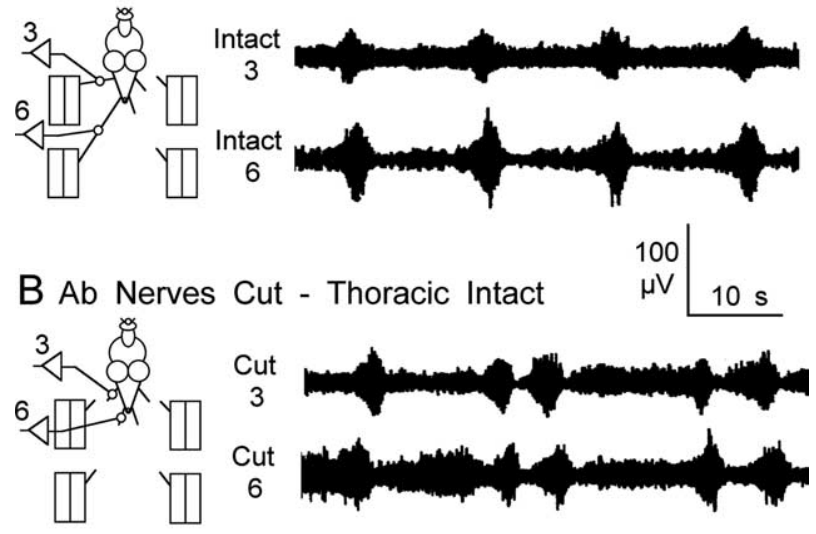

C Brain and Mouth hook

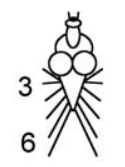

3

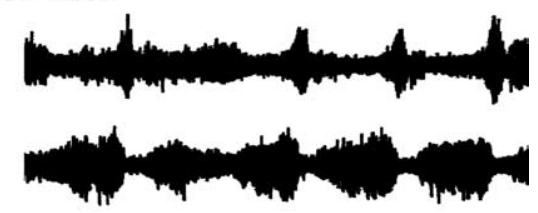

Brain alone

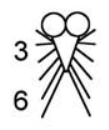

3

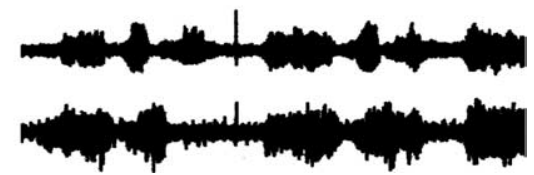

Figure 6. A role of peripheral input in generating the regular rhythmic motor patterns in WT larvae. $\boldsymbol{A}$, Unilateral recordings from anterior segmental nerve 3 and posterior nerve 6 revealed that cutting the nerves to all of the abdominal segments on the contralateral side had little effect on the bursting pattern. $\boldsymbol{B}$, Activity was still organized into bursts when all of the abdominal nerves were cut, but the bursts were not as regular. $C, D$, Reducing peripheral input further by separating the brain from the body wall did not eliminate the burst-like activity. Unilateral recordings were made from anterior segmental nerve 3 and posterior nerve 6 . Preparations consisting of the brain, pharyngeal muscles, and mouth hooks $(\boldsymbol{C})$ or the isolated brain alone $(\boldsymbol{D})$ had periods in which the activity was organized into bursts and showed an increase in tonic activity. L, Left; R, right; Ab, abdominal.

that preparations consisting of the brain, pharyngeal muscles, and mouth hooks or only the isolated brain still had burst-like activities. However, the frequency and regularity of the bursts were reduced and tonic activity increased in these isolated brain preparations (Fig. 6C,D). In summary, bursts persisted in the isolated CNS, suggesting that bursting activity of the motor neuron can be generated without peripheral sensory input. However, the timing of the bursts was irregular, indicating that sensory feedback via segmental nerves is important for producing the regular rhythmic motor pattern controlling larval crawling.

Nerve and muscle activity are correlated

Recordings from the segmental nerve contained spike activities of motor axons that innervate the various muscle fibers, as well as action potentials generated by sensory neurons within the hemisegment. We investigated how activities of the muscle fibers correlate with signals recorded from segmental nerves. Simultaneous recordings were performed using a glass focal patch electrode (Kurdyak et al., 1994) to detect muscle activity and a suction pipette for monitoring segmental nerve spike activities (Fig. $7 A$ ). The patch electrode pressed against the nerve entry point on the muscle surface detected local excitatory junctional currents (EJCs) and thus allowed us to compare the activity of the motor 
A

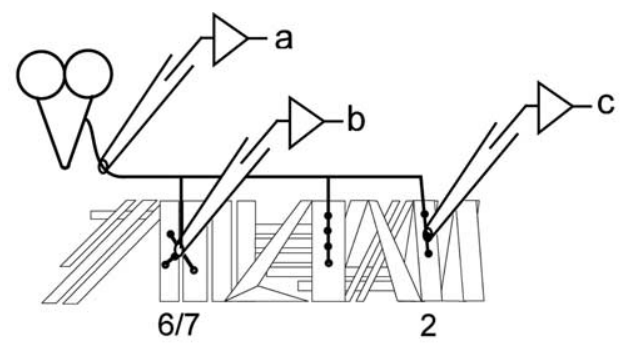

B

$6 / 7$

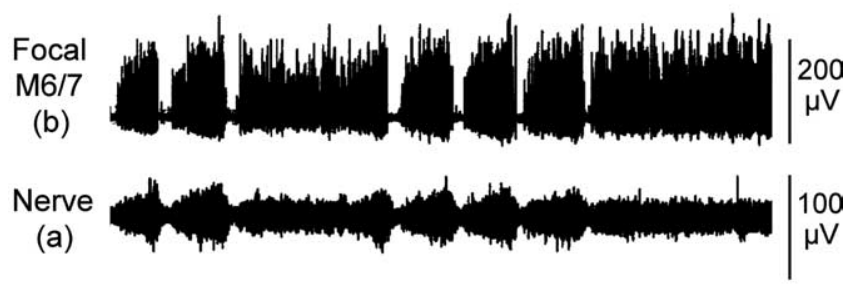

Focal

M2

(c)

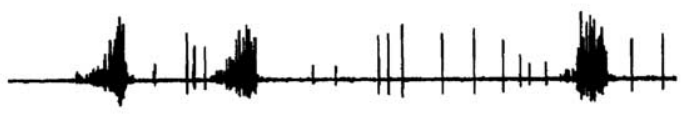

Nerve

(a)

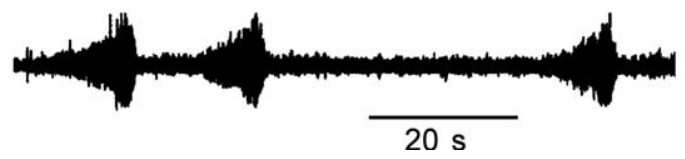

Figure 7. Synaptic activity recorded focally from motor terminals regions on muscles corresponds to activity recorded from nerves. $A$, Diagram of body-wall muscles within an abdominal hemisegment showing focal electrode placement. $\boldsymbol{B}$, Simultaneous en passant recordings from segmental nerves and focal recordings from the nerve entry points of dorsal (M2) or ventral (M6/7) muscles indicated that nerve and muscle activity was coordinated and in phase. Note that the activity recorded from an individual muscle accounts for only part of the activity recorded from the nerve.

neurons that innervate a specific muscle fiber to the ensemble activity from the motor and sensory neurons in the segmental nerve that innervates the hemisegment. These experiments were performed in $0.5 \mathrm{mM} \mathrm{Ca}^{2+}$ saline to reduce muscle contractions and prolong the duration of the focal recordings. Figure 7 shows examples of simultaneous recordings from ventral muscle $6 / 7$ or dorsal muscle 2 compared with the spike activities of the segmental nerve. It was clear that temporally correlated patterns of nerve and muscle activities could be observed in simultaneous recordings, regardless of the muscle fibers within the hemisegment. Both muscles $6 / 7$ and 2 were active during the bursts recorded from the segmental nerve. Notably, bursting activity in the segmental nerve reflected clustered EJCs in muscle focal activity, and these grouped activities for both muscle and nerve tended to end at the same time. No bursting activity was focally recorded from muscles in which the segmental nerve was cut. These results demonstrate that bursting activity detected in the segmental nerve reliably predicted clustered EJCs that drive muscle contraction.

\section{$\boldsymbol{T} \boldsymbol{\beta} \boldsymbol{h}^{n M 18}$ mutants produce fewer rhythmic bursts}

The coordination of nerve spiking activities was compared bilaterally within a single segment and unilaterally between anterior and posterior segments for $T \beta h^{n M 18}$ mutants (Fig. 8). The most striking difference between the $T \beta h^{n M 18}$ mutants and WT larvae was that bursts associated with waves of body-wall contractions were rare in $T \beta h^{n M 18}$ mutants (compare Figs. 3, 8). The locomotive contraction waves were often isolated, not part of a regular rhythmic pattern (Fig. 8). Only occasional intersegmentally coordinated bursts were seen that generated $\mathrm{P}$ to $\mathrm{A}$ ( or A to P)
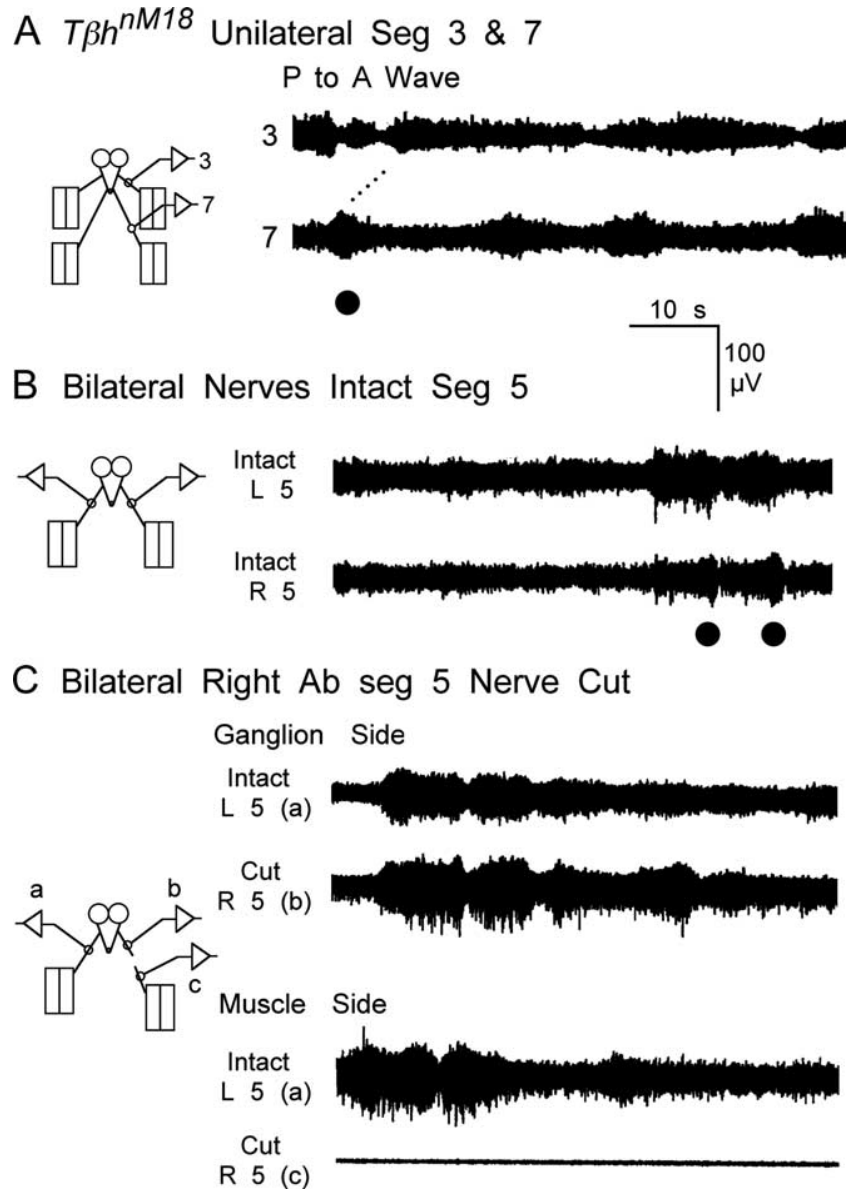

Figure 8. Disruption of locomotor rhythm in $T \beta h^{n M 18}$ mutants with a drastic decrease in rhythmic bursting activity and waves of contractions. $A$, Activity recorded unilaterally from two segmental nerves. There was a phase delay in activity recorded from anterior nerve 3 and posterior nerve 7. Note the increased tonic firing, fewer bursts, and fewer waves. $\boldsymbol{B}$, Activity recorded bilaterally from segment 5 was coordinated and in phase. $C$, Cutting the nerve had little effect on the bursting activity recorded from the proximal stump attached to the ventral ganglion but eliminated activity in the distal stump connected to the body wall, indicating that central neurons produce patterned activity recorded from segmental nerves of $T \beta h^{n M 18} \mathrm{mu}$ tants. L, Left; R, right; Seg, segment; Ab, abdominal.

contraction waves, consistent with the lack of linear locomotion pattern in $T \beta h^{n M 18}$ mutant larvae (Fig. 1) (Saraswati et al., 2004). Most bursts were associated with isolated, local, nonpropagated contractions (Figs. 3, nonrhythmic, 8). For bursts associated with propagating waves, there were no obvious differences from WT in the coordination of activities recorded bilaterally within a single segment or unilaterally from nerves innervating anterior and posterior segments (Fig. 8) (i.e., bilateral recordings were synchronized, and there was a delay in the bursts recorded from nerves innervating anterior and posterior segments).

Similar to the case for WT larvae, surgical manipulation demonstrated that the patterned neuronal activity recorded from the segmental nerves of $T \beta h^{n M 18}$ mutants was generated in the CNS and mainly consisted of the activity of motor neurons (compare Figs. 5, 8). When all of the nerves were intact, a similar bursting pattern was observed for both nerves innervating segment 5 (Fig. $8 B$ ). After cutting the nerve to the right side of segment 5 , the bursting activity continued in the nerve stump attached to the ventral ganglion and disappeared in the distal stump connected to the body wall (Fig. 8C). 
A Bursts

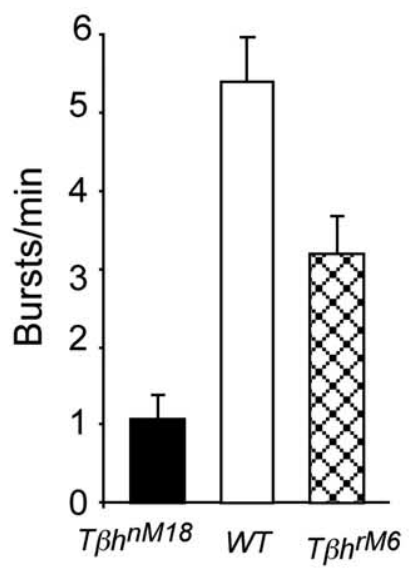

B Strides

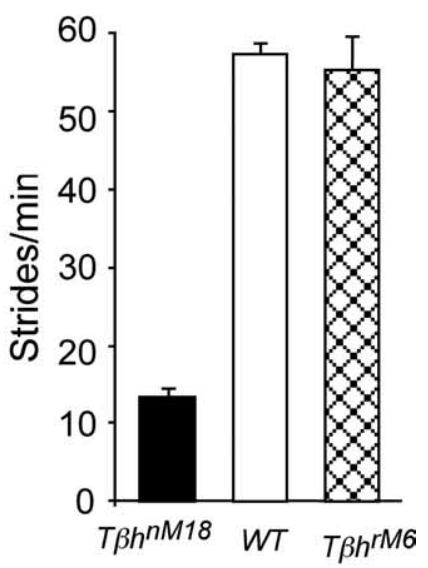

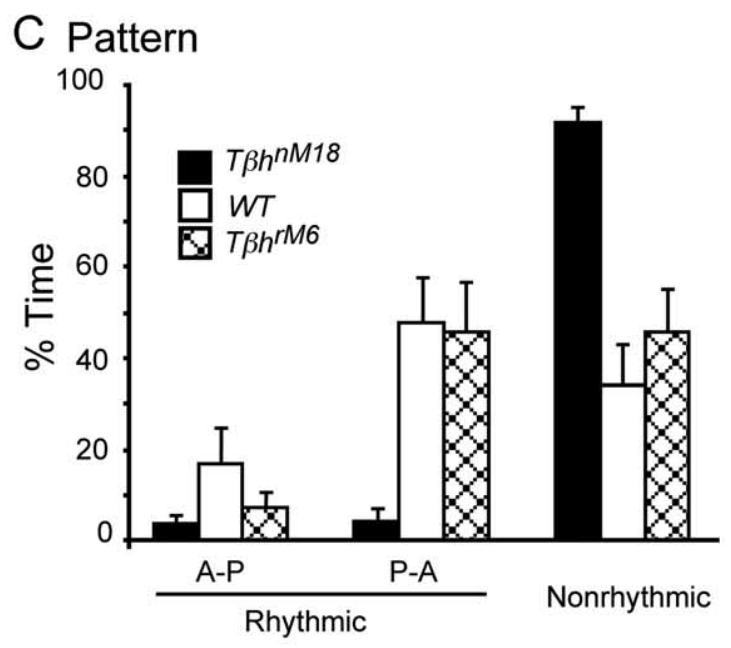

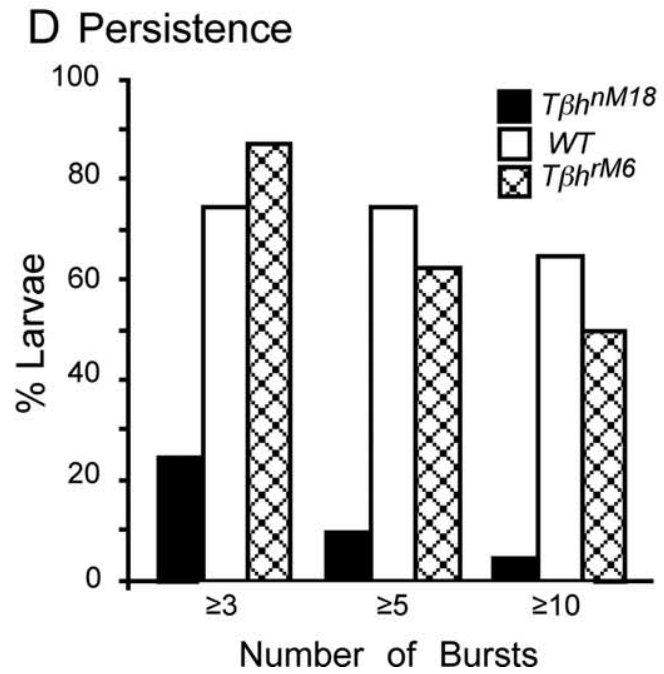

Figure 9. Burst frequency and the persistence of bursting episodes are reduced in $T \beta h^{n M 18}$ mutants. $\boldsymbol{A}$, Summary of the number of bursts produced by WT, $T \beta h^{r M 6}$ revertants, and $T \beta h^{n M 18}$ larvae. All of the bursts associated with waves were counted regardless of the direction of propagation from $T \beta h^{n M 18}$ mutants (20 larvae, $\sim 200 \mathrm{~min}$ ), WT larvae (20 larvae, $\sim 200$ $\mathrm{min}$ ), and $T \beta h^{r M 6}$ revertants (9 larvae, $\sim 50 \mathrm{~min}$ ). $T \beta h^{n M 18}$ mutants had $80 \%$ fewer bursts per minute than the WT larvae and $65 \%$ fewer than $T \beta h^{r M 6}$ revertants $(p<0.001$; Student's $t$ test). $\boldsymbol{B}$, Summary of the number of strides produced by intact WT and $T \beta h^{n M 18}$ larvae crawling on agarose plates. $T \beta h^{n M 18}$ mutants had $\sim 80 \%$ fewer strides per minute than both the WT larvae and $T \beta h^{r M 6}$ revertants (WT, $\left.n=47 ; T \beta h^{n M 18}, n=79 ; T \beta h^{r M 6}, n=19\right)$. Stride data are
The most common activity recorded from WT larvae was a regular rhythmic bursting pattern associated with posterior to anterior waves of contractions. Unlike WT larvae, nonrhythmic activity that was not associated with regular waves of body-wall contractions was more frequent for $T \beta h^{n M 18}$ mutants that have reduced $\mathrm{OA}$ and increased TA levels. We quantified the number of consecutive bursts associated with contraction waves in $T \beta h^{n M 18}$ mutants and larvae with normal levels of OA and TA (i.e., the $T \beta h^{r M 6}$ revertants and WT larvae) (Fig. 9A). All of the bursts were counted regardless of the direction of propagation for larvae from each genotype. On average, $T \beta h^{n M 18}$ mutants had $80 \%$ fewer bursts per minute than the WT larvae and $65 \%$ fewer bursts than the $T \beta h^{r M 6}$ revertants (Fig. 9A). Interestingly, these results resemble the data on the number of strides taken by crawling larvae despite the fact that the number of strides is 10 times lower in the semi-intact preparation (Fig. 9B) (Saraswati et al., 2004).

In addition to having fewer bursts associated with contraction waves, bursts in $T \beta h^{n M 18}$ mutants are less likely to be part of a regular rhythmic pattern. The duration of regular rhythmic bouts of activities associated with locomotive contraction waves and nonrhythmic activities associated with local twitches was measured for $T \beta h^{n M 18}$ mutants, $T \beta h^{r M 6}$ revertants, and WT larvae (Fig. 9C). Using a criterion of bouts that consisted of three or more bursts with a cycle period of $<15 \mathrm{~s}$, we found that $T \beta h^{n M 18}$ mutants expressed nonrhythmic bursting activities significantly more often than $T \beta h^{r M 6}$ revertants and WT larvae. $T \beta h^{n M 18} \mathrm{mu}-$ tants spent $93.6 \pm 3.5 \%$ of the time (average \pm SEM) in nonrhythmic activities, whereas they were observed only $34.4 \pm 9.5 \%$ of the time for WT larvae and $46.6 \pm 9.9 \%$ of the time for $T \beta h^{r M 6}$ revertants (Fig. 9C). T $\beta h^{n M 18}$ mutants spent only $\sim 7 \%$ of the time producing regular rhythmic patterns, and the time was divided equally between $\mathrm{P}$ to $\mathrm{A}$ and $\mathrm{A}$ to $\mathrm{P}$ locomotive contraction waves. In contrast, WT larvae and $T \beta h^{r M 6}$ revertants were much more likely to generate $\mathrm{P}$ to A locomotive waves (Fig. 9C). The time spent in each of the activity patterns was not statistically different for WT larvae and $T \beta h^{r M 6}$ revertants.

To quantify the maintenance of rhythmic bursting activity, the occurrence bouts with 3 bursts in $<30 \mathrm{~s}, 5$ bursts in $<50 \mathrm{~s}$, and 10 bursts in $<100 \mathrm{~s}$ was determined for $T \beta h^{n M 18}$ mutant, $T \beta h^{r M 6}$ revertant, and WT larvae (Fig. 9 D). For WT larvae and $T \beta h^{r M 6}$ revertants, bursting activities commonly persisted for $>5$ bursts and often $>10$ bursts, whereas it was very rare for $T \beta h^{n M 18}$ mutants. In summary, neuronal activity recorded from segmental nerves of $T \beta h^{n M 18}$ mutants appeared to be bilaterally coordinated, but bursts of activity associated with coordinated waves of contractions were rare in these mutants. These results indicate that the locomotion deficit of $T \beta h^{n M 18}$ mutants may be as a result of an altered motor output generated by central neurons.

\section{$\leftarrow$}

from Saraswati et al. (2004). C, Summary of time spent in anterior to posterior waves (A-P), posterior to anterior waves $(\mathrm{P}-\mathrm{A})$, or nonrhythmic bursting unassociated with rhythmic propagating contractions. $T \beta h^{n M 18}$ mutants spent significantly more time producing nonrhythmic contractions than WT larvae or $T \beta h^{r M 6}$ revertants $(p<0.001)$. Note that WT larvae $(n=20)$ and $T \beta h^{r M 6}$ revertants $(n=9)$ generated more P-A waves than A-P waves $(p<0.01)$, whereas time was evenly distributes between A-P and P-A waves for $T \beta h^{n M 18}$ mutants ( $p>$ $0.46, n=20$ ). There was no statistical difference for WT larvae or $T \beta h^{r M 6}$ revertants in the time spent among the patterns. Data were compiled for a 3 min period from each larva. $D$, Summary of the number of episodes that consisted of at least 3,5, and 10 bursting events associated with contraction waves produced by WT, $T \beta h^{r M 6}$ and $T \beta h^{n M 18}$ larvae. $T \beta h^{n M 18}$ mutants $(n=20)$ produced significantly less bursts per episode than WT $(n=20)$ and $T \beta h^{r M 6}(n=8)$ larvae (WT: $p<0.001$ for 3, 5 , and 10 bursts; $T \beta h^{r M 6}: p<0.001$ for 3 bursts, $p<0.01$ for 5 bursts, $p<$ 0.05 for 10 bursts; $t$ test). Data plotted in $\boldsymbol{A}-$ C are average \pm SEM. 


\section{Coordination of activity within a single hemisegment}

To determine how the spike activities of various motor axons are temporally coordinated within a segmental nerve, we investigated the pattern of activation of dorsal, lateral, and ventral muscle fibers within a single abdominal hemisegment for both WT larvae and $T \beta h^{n M 18}$ mutants. To isolate the activity from specific subsets of motor neurons that innervate individual muscle fibers, spontaneous EJCs were recorded simultaneously from different muscles using glass focal patch electrodes pressed against their nerve entry points. Figure 10 shows examples of EJCs collected from ventral muscle 6/7 compared with those recorded simultaneously from lateral muscle 4 or dorsal muscle 2 . It is evident that EJCs recorded from all three muscles were organized into a bursting-like pattern for both WT larvae and $T \beta h^{n M 18}$ mutants (Fig. $10 B, C$ ). These bursts were composed of events of different amplitude (Kurdyak et al., 1994), consistent with previous anatomical studies that each of the muscles is innervated by more than one motor neuron (Sink and Whitington, 1991; Landgraf et al., 1997; Hoang and Chiba, 2001). In general, the activity pattern of clustered EJCs was similar for simultaneous recordings from muscle pairs (e.g., ventral muscle $6 / 7$ with lateral muscle 4 and muscle 6/7 with dorsal muscle 2) (Fig. $10 B, C$ ). However, we noticed that the temporal coordination of the muscle pairs appeared to be tighter in WT larvae than $T \beta h^{n M 18}$ mutants. It is worth noting that the activity in muscle $6 / 7$ tended to precede activity in the other muscles for WT larvae and $T \beta h^{n M 18}$ mutants, suggesting that the contractions begin in ventral muscles and proceed to dorsal muscles.

$\boldsymbol{T} \boldsymbol{\beta} \boldsymbol{h}^{n M 18}$ mutants are capable of generating rhythmic patterns after sensory stimulation

To determine how sensory stimuli affect the motor pattern, WT larvae and $T \beta h^{n M 18}$ mutants were lightly tapped with a silver wire probe on their ventral midline. Tapping the ventral midline of WT larvae near the head generally produced a burst of spike activity in anterior segmental nerves, concurrent with a local contraction of anterior body segments that was not associated with a propagating wave (19 of 20 larvae). This was true regardless of the nerve activity pattern recorded before the tactile stimulation. The anterior contraction was usually followed by a series alternating bursts, originating in anterior segments and terminating in the posterior, that were associated with propagating anterior to posterior locomotive waves (17 of 20 larvae). These anterior to posterior waves were initiated in larvae that were not producing any contraction waves before the stimulus (12 of 13 larvae). In addition, the direction of propagation reversed in most of the larvae producing posterior to anterior waves before being touched (five of seven larvae), and in the remaining larvae, the posterior to anterior locomotive waves resumed after the anterior contraction with a shorter cycle period.

In the example shown in Figure $11 A$, the ongoing nerve activity recorded from WT larvae, before touching, consisted of bursts originating in segment 7 that were followed by bursts in segment 3 , corresponding to posterior to anterior contraction waves (pretouch). Tapping the ventral midline near the head (arrowhead) stopped spiking activities ending the ongoing burst and contraction wave (Fig. 11 A, head touch). (The arrowhead indicates premature termination of the burst recorded from segment 7.) The tap also initiated a burst in segmental nerve 3, concurrent with a local contraction of anterior body segments that was not associated with a propagating wave (asterisk). Most significantly, in the ensuing propagating waves, the relative timing of the segmental nerve activities and the direction of the contraction waves re-

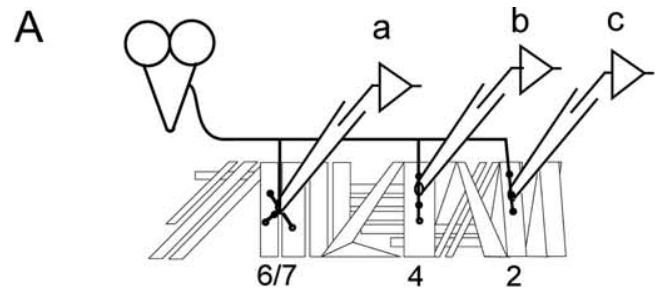

B WT

M4

(b)

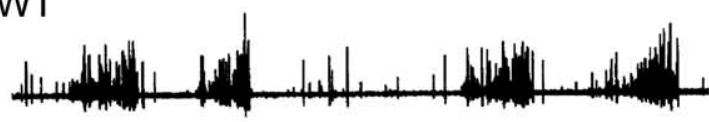

(a)
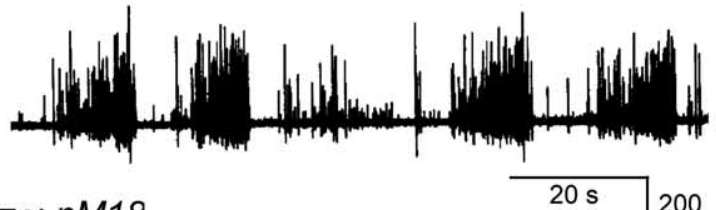

\section{$T \beta h^{n M 18}$}

M4

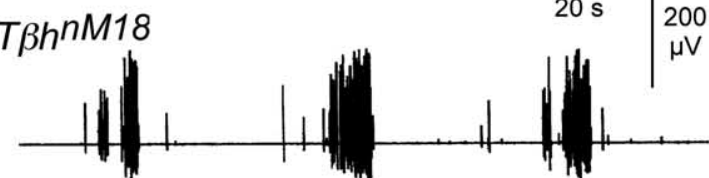

(a)

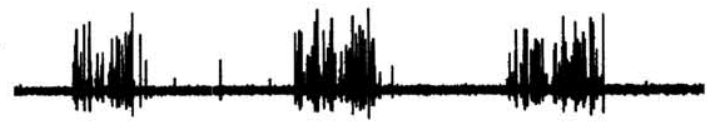

C WT

M2

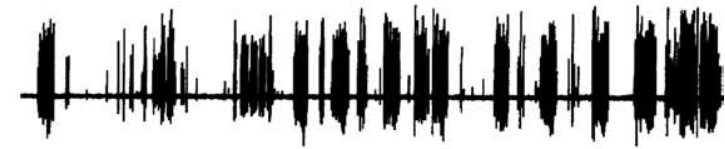

$\mathrm{M} 6 / 7$

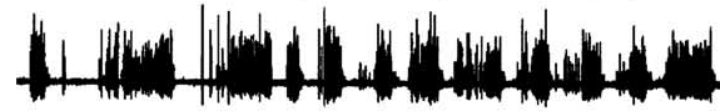

$T \beta h^{n M 18}$

M2

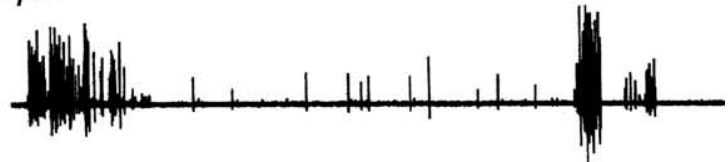

$\mathrm{M} 6 / 7$
(a)

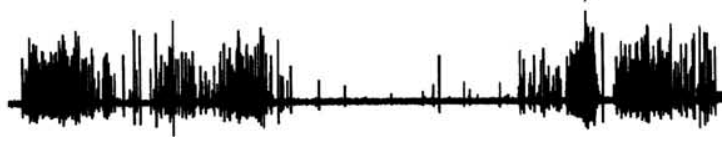

Figure 10. Correlation of activity patterns between dorsal, lateral, and ventral muscles within a hemisegment of WT larvae and $T \beta h^{n M 18}$ mutants. $A$, Diagram of body-wall muscles within an abdominal hemisegment showing focal electrode placement. $\boldsymbol{B}$, Simultaneous focal recording from muscles within the same hemisegment indicated that ventral muscles 6 and 7 (M6/7) and lateral muscle 4 (M4) tend to be activated simultaneously. C, Ventral (M6/7) and dorsal muscle 2 (M2) muscles also have similar activity patterns in both WT larvae and $T \beta h^{n M 18}$ mutants. Despite the concurrent activity observed in each muscle pair, the pattern was similar but not identical within each pair, suggesting that they are innervated by different neurons. Note the weaker correlation of activity between different muscles of $T \beta h^{n M 18}$ mutant larvae.

versed in this WT larvae (i.e., bursts and contractions originated in segment 3 and propagated to segment 7) (Fig. 11 A, head touch). As described above for anterior to posterior waves, the bursts recorded from segmental nerve 3 became longer compared with bursts recorded from the same nerves during waves in the opposite direction.

Tapping the ventral midline near the head of $T \beta h^{n M 18} \mathrm{mu}-$ tants also produced a dramatic effect. The ongoing nerve activity 

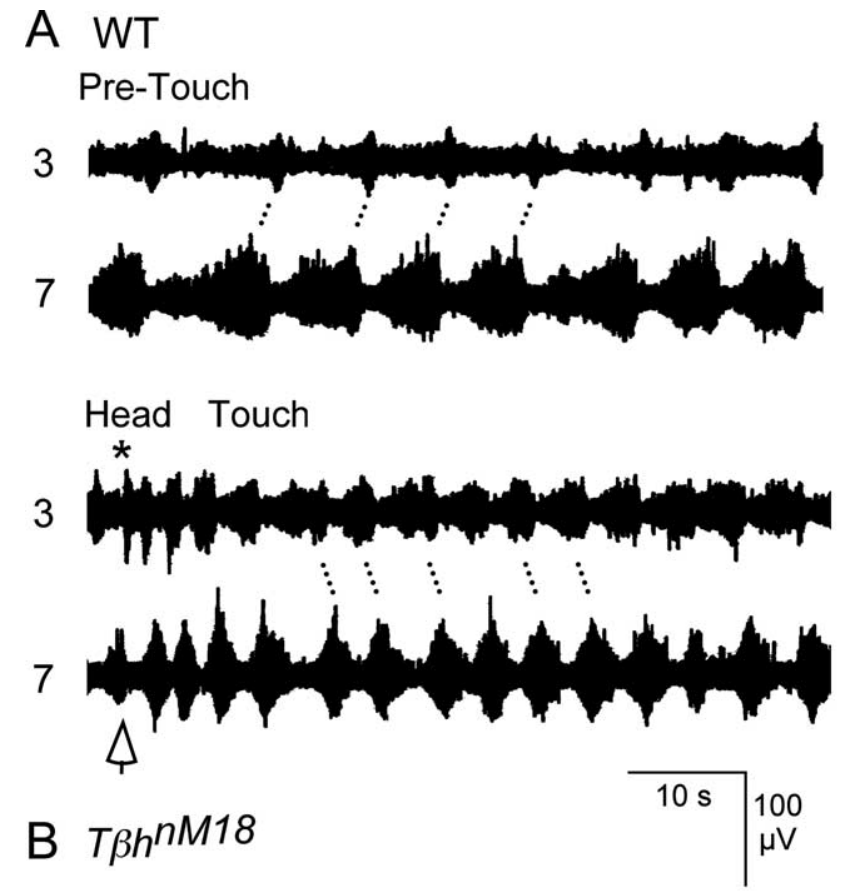

\section{Pre-Touch}

3

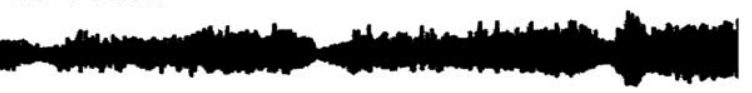

7
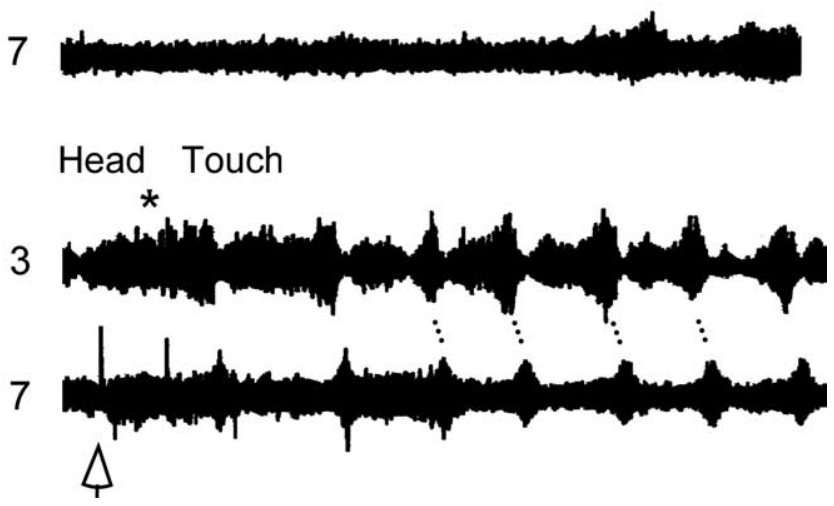

Figure 11. $\quad \boldsymbol{A}, \boldsymbol{B}$, Motor patterns evoked by tactile stimulation in WT larvae $(\boldsymbol{A})$ and $T \beta h^{n M 18}$ mutants $(\boldsymbol{B})$. The anterior end of the larval preparations was lightly tapped with a silver wire probe. Before touching, the ongoing nerve activity consisted of bursts originating in segment 7 that were followed by bursts in segment 3 in WT larvae and nonrhythmic bursting in $T \beta h^{n M 18}$ mutants (pre-touch). Touching the head stopped the ongoing activities (open arrows) and induced a burst observed in segmental nerve 3 that was not associated with a wave (asterisk). The relative timing of the nerve activity reversed for WT larvae, indicating a conversion of posterior to anterior contraction waves to anterior to posterior waves. Significantly, tactile stimulation evoked a series of persistent alternating bursts in segment 3 and 7 of $T \beta h^{n M 18}$ mutants, associated with anterior to posterior waves that were rarely encountered in the spontaneous activity of the mutant larvae.

for all of $T \beta h^{n M 18}$ mutants consisted primarily of nonrhythmic spiking activities that were not associated with contraction waves (11 of 11 larvae) (Fig. $11 \mathrm{~B}$, pre-touch). Touching the head of $T \beta h^{n M 18}$ mutants (arrowhead) produced an extended burst recorded from segmental nerve 3 that was associated with a contraction of the anterior body regions (11 of 11 larvae; asterisk) and not a locomotive contraction wave. Most strikingly, the contraction was followed by alternating bursts, in nerves 3 and 7, that were associated with anterior to posterior waves (8 of 11 larvae) (Fig. 11, head touch). One larva produced posterior to anterior waves in response to tactile stimulation, and two larvae did not produce locomotive waves although they produced anterior body contractions.

Similarly, touching the tail affected both WT larvae and $T \beta h^{n M 18}$ mutants. The stimulus initiated extended contractions and bursts in posterior segments of WT larvae (20 of 20 larvae) and $T \beta h^{n M 18}$ mutants (10 of 11 larvae). This was followed by a rhythmic nerve activity pattern corresponding to contraction waves that proceeded from posterior to anterior body segments ( 18 of 20 for WT larvae; 10 of 11 for $T \beta h^{n M 18}$ mutants).

These bursting activities and the corresponding local contractions and propagating waves are consistent with the behavioral repertoires of intact larvae in response to head or tail tapping. Tactile stimulation first produced local spike activity leading to contractions of the region touched, perhaps reflecting withdrawals in intact larvae. Subsequently, rhythmic waves were generated, propagating from the touched region, perhaps representing locomotion away from the stimulus. These results also demonstrate that the CNS of $T \beta h^{n M 18}$ mutants was capable of generating the motor program necessary for locomotion within a short period after sensory stimulation.

\section{Discussion}

This study presents an analysis of the physiological bases of locomotion control underlying larval crawling behavior using recordings from segmental nerves simultaneous with observations of locomotive contraction waves from a semi-intact larval preparation. We have shown previously that $T \beta h^{n M 18}$ mutants have locomotion defects, spending significantly less time in forward locomotion and taking fewer strides. Our results demonstrate that central effects of the biogenic amines contribute to a major locomotion phenotype of $T \beta h^{n M 18}$ mutants: drastically reduced rhythmic bursts. However, $T \beta h^{n M 18}$ mutants were capable of generating and maintaining a regular rhythmic motor program for a brief period after sensory stimulation. Together, these results suggest that OA and TA are involved in the initiation and modulation of the coordinated activities among the segmental CPGs for crawling in Drosophila larvae.

\section{CPG localization}

Behavioral and electrophysiological studies of Drosophila larval crawling have shown that it is a regular rhythmic behavior that is controlled by a CPG and is amenable to genetic manipulations (Wang et al., 1997, 2002, 2004; Cooper and Neckameyer, 1999; Barclay et al., 2002; Suster and Bate, 2002; Suster et al., 2004; Saraswati et al., 2004). These studies also suggest that the neurons that constitute the CPG and generate the rhythmic pattern for larval locomotion are located in the ventral ganglia of the larval CNS. For example, bursting activity could be pharmacologically evoked from a semi-intact preparation in which the brain lobes were severed from the ventral ganglia (Cattaert and Birman, 2001). In addition, subsets of neurons in the Drosophila brain were shown to contribute to initiation or modulation of locomotion (Suster et al., 2003).

Our results further delineated the localization of the CPG for larval crawling. First, activity recorded en passant from the segmental nerves continued only in the proximal, but not distal, nerve stumps after severing the segmental nerve from the ventral ganglion. We also found that severing all of the nerves to abdominal segments eliminated the locomotive waves but patterned bursting activity continued in the proximal nerve stumps. Finally, the isolated CNS continued to produce some rhythmic burst-like activity. Such nerve activity was clearly not normal in 
that the bursting pattern was not as regular as patterns generated when the brain and body wall were connected. Therefore, it appears that the CPG needs the sensory input to coordinate and maintain the patterned activity and locomotive waves. This interpretation is supported by locomotion studies of Drosophila embryos and larvae in which sensory function was genetically eliminated. Although the CPG for locomotion still develops in the absence of sensory input, forward locomotive waves are significantly reduced (Suster and Bate, 2002). Sensory input is also believed to be important for determining cycle period or segmental coordination in leech and lamprey swimming (Friesen and Cang, 2001).

\section{Intrasegmental coordination}

The coordination of Drosophila larval locomotion is different from locomotion of several other, well studied, limbless organisms such as leeches, earthworms, or caterpillars. Crawling of leeches and earthworms consists of alternating contractions of circular muscles that produce the segmental elongation and longitudinal muscles that produce segmental shortening (Gray and Lissmann, 1938; Gray et al., 1938; Gardner, 1976; Eisenhart et al., 2000). The body-wall musculature of Drosophila abdominal hemisegments $2-7$ is also organized in a stereotypic pattern of transverse and longitudinal muscle fibers (Crossley, 1978). However, it is unlikely that Drosophila crawling is produced by alternating contractions of transverse and longitudinal muscles. In simultaneous recordings from the segmental nerves and longitudinal muscle fibers, we found that only one burst is associated with each contraction wave and that the longitudinal muscles were active for the entire period of each burst (Figs. 7, 10). These results suggest that the activities of transverse and longitudinal muscles most likely overlap. In addition, inspection of video recordings did not reveal any nonoverlapping activities between these two muscle groups within the same segment during contraction waves.

In the Manduca sexta caterpillar, crawling is produced by a wave of contractions that propagates from posterior to anterior segments similar to Drosophila larvae, but the coordination within a segment is different. In Manduca, contractions begin in dorsal regions and terminate in ventral regions (Dominick and Truman, 1986; Johnston and Levine, 1996; Belanger and Trimmer, 2000). In WT Drosophila larvae, the ventral muscles are activated first, as shown by simultaneous focal recordings from both dorsal and ventral longitudinal muscle fibers (Fig. 10). It is interesting to note that the dorsal to ventral progression of contractions is important for the lifting of segments typical of caterpillar-like crawling and that Drosophila larvae, with their ventral to dorsal progression, show little or no vertical displacement during crawling (Berrigan and Pepin, 1995).

\section{Variability in burst patterns and duration}

A variety of clustered spike activities and burst durations were recorded from semi-intact Drosophila larval preparations during episodes of rhythmic activities associated with contraction waves. This robust phenomenon was observed previously using a variety of techniques including en passant nerve recordings, intracellular muscle fiber recordings, or focal synaptic bouton recordings (Budnik et al., 1990; Cooper and Neckameyer, 1999; Cattaert and Birman, 2001, Barclay et al., 2002; Suster et al., 2004; Wang et al., 2004). Although diverse activity patterns have been described, the variation has not been explained. Our en passant recordings from segmental nerves indicate that one clear source of the variation in the spike activity patterns is the direction of the contraction waves (Figs. 3, 4, 8), consistent with previous intracellular muscle recordings (Cattaert and Birman, 2001). Using simultaneous recordings from anterior and posterior segments, we found that the burst duration depends on the recording site and the mode of contraction waves. The duration of the bursts tended to be longer for the nerves innervating the posterior segments during posterior to anterior waves, whereas they were longer for anterior nerves during anterior to posterior waves (Figs. 3, 4, 11). We also observed periods of nonrhythmic activity with burst-like spike patterns that were interspersed between the episodes of regular rhythmic activities (Fig. 3B, 8C). This nonrhythmic activity was often associated with local body-wall contractions that were not part of propagating contraction waves. It is possible that these nonrhythmic bursts and local muscle contractions contribute to nonrhythmic behaviors such as withdrawals, head waving, or turning, described in previous larval behavioral studies (Kernan et al., 1994; Berrigan and Pepin, 1995; Wang et al., 1997, 2002; Saraswati et al., 2004). Therefore, variation in the burst duration and frequencies observed at different recording sites may reflect the organized repertoire of spike activities in successive segments for different modes of movement control during locomotive behaviors.

The observed cycle period for nerve recordings from the semiintact preparation was longer than the period of contraction waves from freely crawling intact larvae. However, the average cycle period from our nerve recordings was within the range of cycle periods reported previously for semi-intact Drosophila larval preparations from other laboratories (Cattaert and Birman, 2001; Barclay et al., 2002; Suster et al., 2004; Wang et al., 2004). Interestingly, it was close to the cycle period for contraction waves from intact larvae that were restrained by pinning their head and tail (Suster et al., 2004).

\section{Modulation of CPG activity by OA and TA}

Previous studies have shown that OA and TA have peripheral effects in Drosophila larvae and adults. Application of both amines to the larval neuromuscular junction modulates the strength of synaptic transmission (Kutsukake et al., 2000; Nagaya et al., 2002). Peripheral effects of OA and its receptor also contribute to an ovulation phenotype in both $T \beta h^{n M 18}$ mutants (deficient in OA) (Monastirioti, 2003) and flies with defective OA receptors (oamb mutants) (Lee et al., 2003).

We have shown that, in addition to their peripheral effects, $\mathrm{OA}$ and TA act on the central neurons that generate the motor program for larval locomotion. OA is known to initiate or modulate the CPG for rhythmic behaviors in other insects, leeches, and earthworms (Hashemzadeh-Gargari and Friesen, 1989; Rameriz and Pearson, 1991; Roeder, 1999; Mizutani et al., 2002). $T \beta h^{n M 18}$ mutants, with elevated TA levels but deficienct in OA (Monastirioti et al., 1996; McClung and Hirsh, 1999), rarely displayed bursts associated with muscle contraction waves (Figs. 8, 9), a defective spike activity pattern apparently reflecting the lack of linear locomotion episodes (Saraswati et al., 2004). However, the CPGs for larval crawling in $T \beta h^{n M 18}$ mutants appeared to retain certain basic function because they were capable of generating a near-normal bursting pattern in response to sensory stimulation. Preliminary physiological experiments indicated that bath application of OA alone produced variable effects on the pattern. This is consistent with our previous behavioral studies suggesting that the phenotype of $T \beta h^{n M 18}$ mutants is likely attributable to an imbalance of OA and TA levels (Saraswati et al., 2004). Together, our observations support the idea that OA and TA are involved in the initiation and modulation of CPG activity. 


\section{Conclusion}

The locomotion deficit of $T \beta h^{n M 18}$ mutants is at least partially attributable to modulatory effects of OA and TA on CPG activity. These results are based on paired, simultaneous recordings of neuronal activity from different segmental nerves in relation to the observed locomotive contraction waves. This simple, straightforward, but incisive approach can be used to examine alterations in the locomotion motor program of other mutants or to study neuromuscular control of additional larval behaviors.

\section{References}

Alkema MJ, Hunter-Ensor M, Ringstad N, Horvitz HR (2005) Tyramine functions independently of octopamine in the Caenorhabditis elegans nervous system. Neuron 46:247-260.

Baier A, Wittek B, Brembs B (2002) Drosophila as a new model organism for the neurobiology of aggression? J Exp Biol 205:1233-1240.

Barclay JW, Atwood HL, Robertson RM (2002) Impairment of central pattern generation in Drosophila cysteine string protein mutants. J Comp Physiol A Neuroethol Sens Neural Behav Physiol 188:71-78.

Belanger JH, Trimmer BA (2000) Combined kinematic and electromyographic analyses of proleg function during crawling by the caterpillar Manduca sexta. J Comp Physiol A Neuroethol Sens Neural Behav Physiol 186:1031-1039.

Berrigan D, Pepin DJ (1995) How maggots move: allometry and kinematics of crawling in larval diptera. J Insect Physiol 41:329-337.

Broadie KS, Bate M (1993) Development of the embryonic neuromuscular synapse of Drosophila melanogaster. J Neurosci 13:144-166.

Buchner E (1991) Genes expressed in the adult brain of Drosophila and effects of their mutations on behavior: a survey of transmitter- and second messenger-related genes. J Neurogenet 7:153-192.

Budnik V, Zhong Y, Wu CF (1990) Morphological plasticity of motor axons in Drosophila mutants with altered excitability. J Neurosci 10:3754-3768.

Buhot MC, Wolff M, Benhassine N, Costet P, Hen R, Segu L (2003) Spatial learning in the 5-HT1B receptor knockout mouse: selective facilitation/ impairment depending on the cognitive demand. Learn Mem 10:466-477.

Cattaert D, Birman S (2001) Blockade of the central generator of locomotor rhythm by noncompetitive NMDA receptor antagonists in Drosophila larvae. J Neurobiol 48:58-73.

Cooper RL, Neckameyer WS (1999) Dopaminergic modulation of motor neuron activity and neuromuscular function in Drosophila melanogaster. Comp Biochem Physiol B 122:199-210.

Crossley CA (1978) The morphology and development of the Drosophila muscular system. In: The genetics and biology of Drosophila, Vol 2b (Ashburner M, Wright TRF, eds), p 449-559, New York: Academic.

Dominick OS, Truman JW (1986) The physiology of wandering behavior in Manduca sexta. III. Organization of wandering behavior in the larval nervous system. J Exp Biol 121:115-132.

Eisenhart FJ, Cacciatore TW, Kristan WB (2000) A central pattern generator underlies crawling in the medicinal leech. J Comp Physiol A Neuroethol Sens Neural Behav Physiol 186:631-643.

Fox LE, Ueda A, Berke B, Peng IF, Wu CF (2004) Movement disorders in Drosophila mutants of potassium channels and biogenic amine pathways. In: Animal models of movement disorders (LeDoux MS, ed), pp 487-504. San Diego: Academic/Elsevier.

Friesen WO, Cang J (2001) Sensory and central mechanisms control intersegmental coordination. Curr Opin Neurobiol 11:678-683.

Gainetdinov RR, Sotnikova TD, Caron MG (2002) Monoamine transporter pharmacology and mutant mice. Trends Pharmacol Sci 23:367-373.

Gardner CR (1976) The neuronal control of locomotion in the earthworm. Biol Rev Camb Philos Soc 51:25-52.

Gray J, Lissmann HW (1938) Studies in animal locomotion. VII. Locomotory reflexes in the earthworm. J Exp Biol 15:506-516.

Gray J, Lissmann HW, Pumphrey RJ (1938) The mechanism of locomotion in the leech (Hirudo medicinalis Ray). J Exp Biol 15:408-430.

Grillner S (1999) Bridging the gap - from ion channels to networks and behaviour. Curr Opin Neurobiol 9:663-669.

Grillner S (2003) The motor infrastructure: from ion channels to neuronal networks. Nat Rev Neurosci 4:573-586.

Hashemzadeh-Gargari H, Friesen WO (1989) Modulation of swimming ac- tivity in the medicinal leech by serotonin and octopamine. Comp Biochem Physiol C 94:295-302.

Hoang B, Chiba A (2001) Single-cell analysis of Drosophila larval neuromuscular synapses. Dev Biol 229:55-70.

Holmes A, Lachowicz JE, Sibley DR (2004) Phenotypic analysis of dopamine receptor knockout mice: recent insights into the functional specificity of dopamine receptor subtypes. Neuropharmacology 47:1117-1134.

Jan LY, Jan YN (1976) Properties of the larval neuromuscular junction in Drosophila melanogaster. J Physiol (Lond) 262:189-214.

Johnston RM, Levine RB (1996) Locomotory behavior in the hawkmoth Manduca sexta: kinematic and electromyographic analyses of the thoracic legs in larvae and adults. J Exp Biol 199:759-774.

Kernan M, Cowan D, Zuker C (1994) Genetic dissection of mechanosensory transduction: mechanoreception-defective mutations of Drosophila. Neuron 12:1195-1206.

Kidokoro Y, Nishikawa K (1994) Miniature endplate currents at the newly formed neuromuscular junction in Drosophila embryo and larvae. Neurosci Res 19:143-154.

Kurdyak P, Atwood HL, Stewart BA, Wu CF (1994) Differential physiology and morphology of motor axons to ventral longitudinal muscles in larval Drosophila. J Comp Neurol 350:463-472.

Kutsukake M, Komatsu A, Yamamoto D, Ishiwa-Chigusa S (2000) A tyramine receptor gene mutation causes a defective olfactory behavior in Drosophila melanogaster. Gene 245:31-42.

Landgraf M, Bossing T, Technau GM, Bate M (1997) The origin, location, and projections of the embryonic abdominal motoneurons of Drosophila. J Neurosci 17:9642-9655.

Lee HG, Seong CS, Kim YC, Davis RL, Han KA (2003) Octopamine receptor OAMB is required for ovulation in Drosophila melanogaster. Dev Biol 264:179-190.

Lesch KP, Zeng Y, Reif A, Gutknecht L (2003) Anxiety-related traits in mice with modified genes of the serotonergic pathway. Eur J Pharmacol 480:185-204.

MacKay-Lyons M (2002) Central pattern generation of locomotion: a review of the evidence. Phys Ther 82:69-83.

Marder E, Buchner D (2001) Central pattern generators and the control of rhythmic movements. Curr Biol 11:R986-R996.

Marder E, Calabrese RL (1996) Principles of rhythmic motor pattern generation. Physiol Rev 76:687-717.

McClung C, Hirsh J (1999) The trace amine tyramine is essential for sensitization to cocaine in Drosophila. Curr Biol 9:853-860.

Mizutani K, Ogawa H, Saito J, Oka K (2002) Fictive locomotion induced by octopamine in the earthworm. J Exp Biol 205:265-271.

Monastirioti M (2003) Distinct octopamine cell population residing in the CNS abdominal ganglion controls ovulation in Drosophila melanogaster. Dev Biol 264:38-49.

Monastirioti M, Linn CE, White K (1996) Characterization of Drosophila tyramine $\beta$-hydroxylase gene and isolation of mutant flies lacking octopamine. J Neurosci 16:3900-3911.

Murchison CF, Zhang XY, Zhang WP, Ouyang M, Lee A, Thomas SA (2004) A distinct role for norepinephrine in memory retrieval. Cell 117:131-143.

Nagaya Y, Kutsukake M, Chigusa SI, Komatsu A (2002) A trace amine, tyramine, functions as a neuromodulator in Drosophila melanogaster. Neurosci Lett 329:324-328.

Ouyang M, Hellman K, Abel T, Thomas SA (2004) Adrenergic signaling plays a critical role in the maintenance of waking and in the regulation of REM sleep. J Neurophysiol 92:2071-2082.

Ramirez JM, Pearson KG (1991) Octopamine induces bursting and plateau potentials in insect neurones. Brain Res 549:332-337.

Roeder T (1999) Octopamine in invertebrates. Prog Neurobiol 59:533-561.

Roeder T (2005) Tyramine and octopamine: ruling behavior and metabolism. Annu Rev Entomol 50:447-477.

Saraswati S, Fox LE, Soll DR, Wu CF (2004) Tyramine and octopamine have opposite effects on the locomotion of Drosophila larvae. J Neurobiol 58:425-441.

Sink H, Whitington PM (1991) Location and connectivity of abdominal motoneurons in the embryo and larva of Drosophila melanogaster. J Neurobiol 22:298-311.

Soll DR (1995) The use of computers in understanding how cells crawl. Int Rev Cytol 163:43-104.

Stewart BA, Atwood HL, Renger JJ, Wang J, Wu CF (1994) Improved stability of Drosophila larval neuromuscular preparations in haemolymph- 
like physiological solutions. J Comp Physiol A Neuroethol Sens Neural Behav Physiol 175:179-191.

Suster ML, Bate M (2002) Embryonic assembly of a central pattern generator without sensory input. Nature 416:174-178.

Suster ML, Martin JR, Sung C, Robinow S (2003) Targeted expression of tetanus toxin reveals sets of neurons involved in larval locomotion in Drosophila. J Neurobiol 55:233-246.

Suster ML, Karunanithi S, Atwood HL, Sokolowski MB (2004) Turning behavior in Drosophila larvae: a role for the small scribbler transcript. Genes Brain Behav 3:273-286.

Tempel BL, Livingstone MS, Quinn WG (1984) Mutations in the dopa decarboxylase gene affect learning in Drosophila. Proc Natl Acad Sci USA 81:3577-3581.

Viggiano D, Ruocco LA, Sadile AG (2003) Dopamine phenotype and behaviour in animal models: in relation to attention deficit hyperactivity disorder. Neurosci Biobehav Rev 27:623-637.
Waddell S, Quinn WG (2001) Flies, genes, and learning. Annu Rev Neurosci 24:1283-1309.

Wang JW, Sylwester AW, Reed D, Wu DA, Soll DR, Wu CF (1997) Morphometric description of the wandering behavior in Drosophila larvae: aberrant locomotion in $\mathrm{Na}^{+}$and $\mathrm{K}^{+}$channel mutants revealed by computer-assisted motion analysis. J Neurogenet 11:231-254.

Wang JW, Soll DR, Wu CF (2002) Morphometric description of the wandering behavior in Drosophila larvae: a phenotypic analysis of $\mathrm{K}^{+}$channel mutants. J Neurogenet 16:45-63.

Wang P, Saraswati S, Guan Z, Watkins CJ, Wurtman RJ, Littleton JT (2004) A Drosophila temperature-sensitive seizure mutant in phosphoglycerate kinase disrupts ATP generation and alters synaptic function. J Neurosci 24:4518-4529.

Wu CF, Ganetzky B, Jan LY, Jan YN, Benzer S (1978) A Drosophila mutant with a temperature-sensitive block in nerve conduction. Proc Natl Acad Sci USA 8:4047-4051. 